\title{
MAGNETIC VORTICES FOR A GINZBURG-LANDAU TYPE ENERGY WITH DISCONTINUOUS CONSTRAINT
}

\author{
AYMAN KACHMAR ${ }^{1,2}$
}

\begin{abstract}
This paper is devoted to an analysis of vortex-nucleation for a Ginzburg-Landau functional with discontinuous constraint. This functional has been proposed as a model for vortex-pinning, and usually accounts for the energy resulting from the interface of two superconductors. The critical applied magnetic field for vortex nucleation is estimated in the London singular limit, and as a by-product, results concerning vortex-pinning and boundary conditions on the interface are obtained.
\end{abstract}

Mathematics Subject Classification. 35J60, 35J20, 35J25, 35B40, 35Q55, 82D55.

Received April 30, 2008. Revised February 13, 2009.

Published online June 18, 2009.

\section{INTRODUCTION AND MAIN RESULTS}

It is widely accepted among the physics community that spatial inhomogeneities, impurities or point defects in a superconducting sample provide pinning sites for vortices, preventing thus their motion and the resultant induced resistivity, see $[9,10]$ and the references therein. A similar behavior has also been observed in superconducting samples subject to non-constant temperatures, see [26].

In the framework of the Ginzburg-Landau theory, it is proposed to model the energy of an inhomogeneous superconducting sample by means of the following functional (see [10,27])

$$
\mathcal{G}_{\varepsilon, H}(\psi, A)=\int_{\Omega}\left(|(\nabla-i A) \psi|^{2}+\frac{1}{2 \varepsilon^{2}}\left(p(x)-|\psi|^{2}\right)^{2}+|\operatorname{curl} A-H|^{2}\right) \mathrm{d} x .
$$

Here, $\Omega \subset \mathbb{R}^{2}$ is the 2-D cross section of the superconducting sample, assumed to occupy a cylinder of infinite height. The complex-valued function $\psi \in H^{1}(\Omega ; \mathbb{C})$ is called the 'order parameter', whose modulus $|\psi|^{2}$ measures the density of the superconducting electron Cooper pairs (hence $\psi \equiv 0$ corresponds to a normal state), and the real vector field $A=\left(A_{1}, A_{2}\right)$ is called the 'magnetic potential', such that the induced magnetic field in the sample corresponds to $\operatorname{curl} A$.

The functional (1.1) depends on several parameters: $\frac{1}{\varepsilon}=\kappa$ is a characteristic of the superconducting sample (a temperature independent quantity), $H \geq 0$ is the intensity of the applied magnetic field (assumed constant

\footnotetext{
Keywords and phrases. Generalized Ginzburg-Landau energy functional, proximity effects, global minimizers, unique positive solution, vortices.

${ }^{1}$ Université Paris-Sud, Département de mathématique, Bât. 425, 91405 Orsay, France.

2 Aarhus University, Department of mathematical sciences, 1530 Ny Munkegade, 8000 Aarhus C, Denmark.

ayman.kachmar@math.u-psud.fr
} 
and parallel to the axis of the superconducting sample), $p(x)$ is a positive function modeling the impurities in the sample, whose values are temperature dependent. The positive sign of the function $p$ means that the temperature remains below the critical temperature of the superconducting sample.

It is standard, starting from a minimizing sequence, to prove existence of minimizers of the functional (1.1) in the space $H^{1}(\Omega ; \mathbb{C}) \times H^{1}\left(\Omega ; \mathbb{R}^{2}\right)$, see e.g. [15]. A minimizer $(\psi, A)$ of $(1.1)$ is a weak solution of the G-L equations:

$$
\left\{\begin{array}{l}
-(\nabla-i A)^{2} \psi=\frac{1}{\varepsilon^{2}}\left(p(x)-|\psi|^{2}\right) \psi, \quad \text { in } \Omega \\
\nabla^{\perp} \operatorname{curl} A=(i \psi,(\nabla-i A) \psi) \quad \text { in } \Omega \\
n(x) \cdot(\nabla-i A) \psi=0, \quad \operatorname{curl} A=H \quad \text { on } \partial \Omega
\end{array}\right.
$$

where $n(x)$ is the unite outward normal vector of $\partial \Omega$.

It has been conjectured that for a minimizing configuration $(\psi, A)$ of $(1.1)$, the vortices (zeros of $\psi$ ) should be pinned near the minimal points of the function $p$ (or near the critical points if $p$ is smooth), see [9,27]. Many authors have addressed this question in the regime of extreme type II superconducting materials, $\varepsilon \rightarrow 0$. For instance, Aftalion et al. [1] analyze the situation when $p$ is periodic and smooth, André et al. [5] analyze the situation when $p$ is smooth and having a finite number of isolated zeros, and Alama and Bronsard [3] allow $p$ to have negative values in some normal regions of the sample. A similar functional arises also in the setting of Bose-Einstein condensates, see [2]. We would also like to mention the interesting work of Sigal and Ting [32], who prove existence and uniqueness of solutions with pinned vortices for the Ginzburg-Landau equation (1.2) in $\mathbb{R}^{2}$ when $H=0$ and the potential $p$ is in a suitable class.

In this paper, the function $p$ is a step function. We take $\Omega=D(0,1)$ the unit disc in $\mathbb{R}^{2}$, and

$$
p(x)= \begin{cases}1 & \text { if }|x| \leq R \\ a & \text { if } R<|x|<1\end{cases}
$$

where $a \in \mathbb{R}_{+} \backslash\{1\}$ and $0<R<1$ are given constants.

Putting

$$
S_{1}=D(0, R), \quad S_{2}=D(0,1) \backslash \overline{D(0, R)},
$$

then the above choice of $p$ has two physical interpretations:

- $S_{1}$ and $S_{2}$ correspond to two superconducting samples with different critical temperatures.

- The superconducting sample $\Omega$ is subject to two different temperatures in the regions $S_{1}$ and $S_{2}$, which may happen by cold or heat working $S_{2}$.

Lassoued-Mironescu analyze the functional (1.1) without magnetic field (i.e. $A=0$ and $H=0$ ) and with $p$ as given in (1.3), by assuming that minimizers satisfy a Dirichlet boundary condition, $\psi=g$ on $\partial \Omega$, with $g$ valued in $\mathbb{S}^{1}$ and has degree $d>0$, much in the same spirit of Béthuel et al. [8]. When $a>1$ and $\varepsilon \rightarrow 0$, they obtain that minimizers have $d$ vortices, strictly localized in $S_{1}$, and whose positions are determined by a finite dimensional problem (a renormalized energy).

In this paper, minimization of the functional (1.1) will take place in the space

$$
\mathcal{H}=H^{1}(\Omega ; \mathbb{C}) \times H^{1}\left(\Omega ; \mathbb{R}^{2}\right)
$$

Thus we do not assume a priori boundary conditions for admissible configurations, but minimizers satisfy natural boundary conditions. We study nucleation of vortices as the applied magnetic field varies, and we obtain that their behavior is strongly dependent on the parameter $a$, leading in some situations (small values of $a$ ) to a pinning phenomenon. 
We summarize in the next theorem some of the main results we have obtained concerning the case of small values of $a$.

Theorem 1.1. There exists a constant $\left.a_{0} \in\right] 0,1[$, and for each $a \in] 0, a_{0}\left[\right.$, there exist positive constants $\lambda_{*}$, $\lambda_{\#}, \varepsilon_{0}$ and a function $] 0, \varepsilon_{0}\left[\ni \varepsilon \mapsto k_{\varepsilon} \in \mathbb{R}_{+}, 0<\liminf _{\varepsilon \rightarrow 0} k_{\varepsilon} \leq \limsup _{\varepsilon \rightarrow 0} k_{\varepsilon}<\infty\right.$, such that, if $\left(\psi_{\varepsilon, H}, A_{\varepsilon, H}\right)$ is a minimizer of (1.1), then:

(1) If $H<k_{\varepsilon}|\ln \varepsilon|-\lambda_{*} \ln |\ln \varepsilon|$, then $\left|\psi_{\varepsilon, H}\right| \geq \frac{\sqrt{a}}{2}$ in $\bar{\Omega}$.

(2) If $H=k_{\varepsilon}|\ln \varepsilon|+\lambda \ln |\ln \varepsilon|$ and $\lambda \geq-\lambda_{*}$, then there exists a finite family of balls $\left(B\left(a_{i}(\varepsilon), r_{i}(\varepsilon)\right)\right)_{i \in I}$ with the following properties:

(a) $\sum_{i \in I} r_{i}(\varepsilon)<|\ln \varepsilon|^{-10}$.

(b) $\left|\psi_{\varepsilon, H}\right| \geq \frac{\sqrt{a}}{2}$ in $\Omega \backslash \bigcup_{i \in I} B\left(a_{i}(\varepsilon), r_{i}(\varepsilon)\right)$.

(c) Letting $d_{i}$ be the degree of $\psi_{\varepsilon, H} /\left|\psi_{\varepsilon, H}\right|$ on $\partial B\left(a_{i}(\varepsilon), r_{i}(\varepsilon)\right)$ if $B\left(a_{i}, r_{i}\right) \subset \Omega$ and 0 otherwise, then we have

$$
\sup _{\substack{i \in I \\\left|d_{i}\right|>0}}|R-| a_{i}(\varepsilon)|| \rightarrow 0 \quad \text { as } \varepsilon \rightarrow 0 .
$$

(d) If $\lambda>\lambda_{\#}$ there exist positive constants $c$ and $C$ independent of $\varepsilon$ such that

$$
\left.\left.c \ln |\ln \varepsilon| \leq \sum_{i}\left|d_{i}\right| \leq C \ln |\ln \varepsilon| \quad \forall \varepsilon \in\right] 0, \varepsilon_{0}\right]
$$

Theorem 1.1 exhibits a completely different regime for the nucleation of vortices when compared with the usual G-L functional defined in a simply connected domain [29], and the result is qualitatively much more in the direction of a circular annulus superconductor/condensate (cf. [2,3]). In particular, Theorem 1.1 states that vortices are localized near the less-superconducting regions of the sample (i.e. $\left.S_{2}\right)$ : This is the pinning phenomenon predicted in the physics literature, see e.g. [9].

In light of Theorem 1.1, let us define, as in [29], the vorticity measure (see Prop. 4.1 for a more canonical definition),

$$
\mu\left(\psi_{\varepsilon, H}, A_{\varepsilon, H}\right)=\sum_{i} d_{i} \delta_{a_{i}}
$$

Theorem 1.1 shows that in the regime (d), we have (up to the extraction of a subsequence)

$$
\frac{\mu\left(\psi_{\varepsilon, H}, A_{\varepsilon, H}\right)}{\ln |\ln \varepsilon|} \rightarrow \mu_{*} \quad \text { as } \varepsilon \rightarrow 0,
$$

where $\mu_{*}$ is a measure supported in the circle $\partial D(0, R)$. We conjecture that $\mu_{*}$ is indeed a constant times the Lebesgue measure.

When $a>1$, we obtain a completely different behavior, which is that of [29]. As in Theorem 1.1, we get $k_{\varepsilon}>0$, and a sequence of 'critical fields'

$$
H_{c, n}(\varepsilon)=k_{\varepsilon}(|\ln \varepsilon|+(n-1) \ln |\ln \varepsilon|), \quad(n \geq 1)
$$

such that, if $H_{n}<H<H_{n+1}$, then for a minimizer $\left(\psi_{\varepsilon}, A_{\varepsilon}\right)$ of $(1.1), \psi_{\varepsilon}$ has exactly $n$ vortices $\left\{x_{i}(\varepsilon)\right\}_{i=1}^{n}$, each of degree 1 , and there positions are determined by minimizing a finite dimensional problem, i.e. a renormalized energy (see Sect. 6.3). 


\section{Boundary conditions}

In addition to the pinning phenomenon, we obtain as a by-product some interpretation concerning the boundary condition on the $S_{1}-S_{2}$ interface. The precise result is the following.

Theorem 1.2. There exists a function $\mathbb{R}_{+} \backslash\{1\} \ni a \mapsto \gamma(a) \in \mathbb{R} \backslash\{0\}$ such that, if $\left(\psi_{\varepsilon, H}, A_{\varepsilon, H}\right)$ is a minimizer of (1.1) satisfying $\left|\psi_{\varepsilon, H}\right|>0$ in $\bar{\Omega}$, then the following limit holds:

$$
\lim _{\varepsilon \rightarrow 0}\left\|\varepsilon \frac{n(x) \cdot\left(\nabla-i A_{\varepsilon, H}\right) \psi_{\varepsilon, H}}{\psi_{\varepsilon, H}}+\gamma(a)\right\|_{L^{2}(\partial D(0, R))}=0
$$

where $n(x)=\frac{x}{|x|}$ for all $x \in \mathbb{R}^{2} \backslash\{0\}$, is the outward unit normal vector.

Furthermore, the function $\gamma$ satisfies: (1) $\gamma(a)>0$ if $a<1$; (2) $\gamma(a)<0$ if $a>1$.

Thus, below the first critical field $H_{C_{1}}$, minimizers approximately satisfy a Robin-type boundary condition on the $S_{1}$-boundary:

$$
n(x) \cdot\left(\nabla-i A_{\varepsilon, H}\right) \psi_{\varepsilon, H}+\frac{\gamma(a)}{\varepsilon} \psi_{\varepsilon, H}(1+o(1))=0 \quad \text { on } \partial S_{1} .
$$

This is a boundary condition of the type predicted by de Gennes $(\gamma(a)$ being called the de Gennes parameter $)$, see [11]. When $a>1, \gamma(a)<0$, hence we justify the modeling of Fink and Joiner [13], who use a negative 'de Gennes parameter' to model a superconductor surrounded by another superconductor with a higher critical temperature. They claim also that this is the setting when cold working the surface of superconducting samples (see [16,26] for more recent reviews of this topic).

The result of Theorem 1.2 also justifies the analysis we carried out in $[17,19,20]$ for problems involving boundary conditions of the type (1.7), and complements results in this direction obtained in [18,22].

Preliminary versions of Theorems 1.1 and 1.2 were announced without proofs in [21]. In [6], the case of general domains and the regime of applied magnetic fields comparable with $|\ln \varepsilon|$ are also treated.

\section{Main points of the proofs}

Let us briefly describe the main points of the proofs of Theorems 1.1 and 1.2, and thus explain what stands behind their statements.

The starting point is the analysis of minimizers of (1.1) when $H=0$. In this case, (1.1) has, up to a gauge transformation, a unique minimizer $\left(u_{\varepsilon}, 0\right)$ where $u_{\varepsilon}$ is a positive real-valued function. The asymptotic profile of $u_{\varepsilon}$ as $\varepsilon \rightarrow 0$ is obtained in Theorem 2.4, which proves Theorem 1.2 when $H=0$ with a stronger convergence in $L^{\infty}$ norm.

When $H>0$, let $(\psi, A)$ be a minimizer of (1.1). Inspired by Lassoued and Mironescu [23], we introduce a normalized density ${ }^{3}$

$$
\varphi=\frac{\psi}{u_{\varepsilon}}
$$

Then $|\varphi| \leq 1$ and we are led to the analysis of the following functional (see Lem. 2.7)

$$
\mathcal{F}_{\varepsilon, H}(\varphi, A)=\int_{\Omega}\left(u_{\varepsilon}^{2}|(\nabla-i A) \varphi|^{2}+\frac{1}{2 \varepsilon^{2}} u_{\varepsilon}^{4}\left(1-|\varphi|^{2}\right)^{2}+|\operatorname{curl} A-H|^{2}\right) \mathrm{d} x
$$

using tools from Sandier and Serfaty [29].

\footnotetext{
${ }^{3}$ Notice that $\varphi$ and $\psi$ have the same vortices.
} 
When we take $\varphi=1$ in (1.8) and we minimize the resulting functional over $A \in H^{1}\left(\Omega ; \mathbb{R}^{2}\right)$, we get that the minimizer is $\frac{H}{u_{\varepsilon}^{2}} \nabla^{\perp} h_{\varepsilon}$, where $\left.h_{\varepsilon}: \Omega \longrightarrow\right] 0,1[$ is the solution of the equation:

$$
-\operatorname{div}\left(\frac{1}{u_{\varepsilon}^{2}} \nabla h_{\varepsilon}\right)+h_{\varepsilon}=0 \quad \text { in } \Omega, \quad h_{\varepsilon}=1 \quad \text { on } \partial \Omega .
$$

The constant $k_{\varepsilon}$ appearing in Theorem 1.1 is defined by

$$
k_{\varepsilon}=\frac{1}{2}\left(\max _{x \in \bar{\Omega}} \frac{1-h_{\varepsilon}(x)}{u_{\varepsilon}^{2}(x)}\right)^{-1} .
$$

Thanks to our choice of the domain $\Omega$ and the step function $p(x)$ in (1.3), we show that the function $h_{\varepsilon}(x)$ is radially symmetric and strictly increasing with respect to $|x|$ (see Lem. 3.1). This permits to show that

$$
0<\liminf _{\varepsilon \rightarrow 0} k_{\varepsilon} \leq \limsup _{\varepsilon \rightarrow 0} k_{\varepsilon}<+\infty .
$$

Roughly speaking, the analysis of Sandier and Serfaty ( $c f .[29])$ says that near the first critical magnetic field, the vortices of a minimizer of ${ }^{4}(1.8)$ are localized as $\varepsilon \rightarrow 0$ near the set $\Lambda_{\varepsilon}=\left\{x \in \bar{\Omega}: \frac{1-h_{\varepsilon}(x)}{u_{\varepsilon}^{2}(x)}=\frac{1}{2} k_{\varepsilon}^{-1}\right\}$. We localize the set $\Lambda_{\varepsilon}$ by means of a fine semi-classical analysis. We obtain when $a$ is sufficiently small that the set $\Lambda_{\varepsilon}$ consists of a circle $\partial B\left(0, R_{\varepsilon}\right)$, where $\left.R_{\varepsilon} \in\right] R, 1[$ has the following asymptotic behavior (see Thm. 3.4):

$$
\varepsilon \ll R_{\varepsilon}-R \ll \varepsilon^{\alpha} \quad \text { as } \varepsilon \rightarrow 0, \quad(\alpha \in] 0,1[\text { is given }) .
$$

Let us mention that when $a>1$, we prove that the set $\Lambda_{\varepsilon}$ consists of a single point, $\Lambda_{\varepsilon}=\{0\}$, and for this reason, minimizers of (1.1) exhibit the same behavior as the one present in [29], i.e. near the first critical magnetic field, a minimizer has a finite number of vortices localized near the center of the disc and whose exact positions are determined by a finite dimensional problem (a renormalized energy).

The rest of the proof is devoted to the construction of matching lower and upper bounds. Using the detailed asymptotic behavior of $h_{\varepsilon}$ as $\varepsilon \rightarrow 0$, we may adapt essentially the same construction of vortex-balls provided by Sandier and Serfaty [29]. For the upper bound, the situation is a bit more delicate, since the function $u_{\varepsilon}$ has a boundary layer behavior and we construct a test configuration with vortices localized near the circle $C(0, R)$. The challenge is to prove that such a configuration carries the right amount of energy. Technically, one does that via a Green's potential $G_{\varepsilon}$ which we were not able to give a good control of it, see Lemma 5.2. To overcome this difficulty, we seek vortices situated on the circle $\gamma=C\left(0, R+\frac{\ln |\ln \varepsilon|}{\ln \varepsilon \mid}\right)$, where a nice $L^{1}$ bound can be shown to hold for $G_{\varepsilon}(x, x)$ on $\gamma$. Then, using a specific result stated and proved in Lemma 5.5, the aforementioned $L^{1}$ bound provides us with a family of well separated points $\left(a_{i}\right)$ serving as the centers of vortices for the test configuration and for which $G_{\varepsilon}\left(a_{i}, a_{i}\right)$ satisfies a nice bound for all $i$, see Lemma 5.4. The proof then goes essentially as $[2,29]$.

\section{Outline of the paper}

Section 2 is devoted to a preliminary analysis of the variational problem (1.1). In particular, a detailed analysis is given for the case without magnetic field $H=0$.

Section 3 is devoted to an analysis of equation (1.9).

Section 4 is devoted to derive a lower bound of the minimizing energy, involving the construction of vortexballs.

Section 5 is devoted to establish an upper bound of the minimizing energy, that is involved with a careful analysis of a Green's potential.

\footnotetext{
${ }^{4}$ These are also the vortices of a minimizer of (1.1).
} 
Finally, Section 6 is devoted to the proofs of Theorems 1.1 and 1.2, through the matching of the lower and upper bounds obtained in Sections 4 and 5 respectively.

$A$ remark on the notation

The letters $C, \widetilde{C}, M$, etc., will denote positive constants independent of $\varepsilon$. For $n \in \mathbb{N}$ and $X \subset \mathbb{R}^{n},|X|$ denotes the Lebesgue measure of $X . B(x, r)$ denotes the open ball in $\mathbb{R}^{n}$ of radius $r$ and center $x .(\cdot, \cdot)$ denotes the scalar product in $\mathbb{C}$ when identified with $\mathbb{R}^{2}$. For two positive functions $a(\varepsilon)$ and $b(\varepsilon)$, we write $a(\varepsilon) \ll b(\varepsilon)$ as $\varepsilon \rightarrow 0$ to mean that $\lim _{\varepsilon \rightarrow 0} \frac{a(\varepsilon)}{b(\varepsilon)}=0$.

\section{Preliminary ANAlysis of Minimizers}

\subsection{The case without applied magnetic field}

This section is devoted to an analysis for minimizers of (1.1) when the applied magnetic field $H=0$. We follow closely similar results obtained in [18].

We keep the notation introduced in Section 1. Upon taking $A=0$ and $H=0$ in (1.1), one is led to introduce the functional

$$
\mathcal{G}_{\varepsilon}(u):=\int_{\Omega}\left(|\nabla u|^{2}+\frac{1}{2 \varepsilon^{2}}\left(p(x)-u^{2}\right)^{2}\right) \mathrm{d} x
$$

defined for functions in $H^{1}(\Omega ; \mathbb{R})$.

We introduce

$$
C_{0}(\varepsilon)=\inf _{u \in H^{1}(\Omega ; \mathbb{R})} \mathcal{G}_{\varepsilon}(u) .
$$

The next theorem is an analogue of Theorem 1.1 in [18].

Theorem 2.1. Given $a \in \mathbb{R}_{+} \backslash\{1\}$, there exists $\varepsilon_{0}$ such that for all $\left.\varepsilon \in\right] 0, \varepsilon_{0}[$, the functional (2.1) admits in $H^{1}(\Omega ; \mathbb{R})$ a minimizer $u_{\varepsilon} \in C^{2}\left(\overline{S_{1}}\right) \cup C^{2}\left(\overline{S_{2}}\right)$ such that

$$
\min (1, \sqrt{a})<u_{\varepsilon}<\max (1, \sqrt{a}) \quad \text { in } \bar{\Omega} .
$$

Furthermore, with our choice of the domains $\Omega, S_{1}$ and $S_{2}$ in (1.4), the function $u_{\varepsilon}$ is radial.

If $H=0$, minimizers of (1.1) are gauge equivalent to the state $\left(u_{\varepsilon}, 0\right)$.

The asymptotic behaviour of the function $u_{\varepsilon}$ when $\varepsilon \rightarrow 0$ is based on the understanding of the following canonical equation:

$$
\left\{\begin{array}{l}
-\Delta u=\left(1-u^{2}\right) u \text { in } \mathbb{R} \times \mathbb{R}_{-}, \quad-\Delta u=\left(a-u^{2}\right) u \text { in } \mathbb{R} \times \mathbb{R}_{+}, \\
\partial_{x_{2}} u\left(\cdot, 0_{-}\right)=\partial_{x_{2}} u\left(\cdot, 0_{+}\right), \quad u\left(\cdot, 0_{-}\right)=u\left(\cdot, 0_{+}\right) \quad \text { on } \mathbb{R} .
\end{array}\right.
$$

When $a \neq 1$, it is easy to verify that (2.3) has the following solution

$$
\mathbb{R}^{2} \ni\left(x_{1}, x_{2}\right) \mapsto U\left(x_{2}\right),
$$

where the function $U\left(x_{2}\right)$ is defined by

$$
U\left(x_{2}\right)=\left\{\begin{array}{l}
\frac{\beta_{1}(a) \exp \left(-\sqrt{2} x_{2}\right)-1}{\beta_{1}(a) \exp \left(-\sqrt{2} x_{2}\right)+1}, \quad \text { if } x_{2} \in \mathbb{R}_{-}, \\
\sqrt{a} \frac{\beta_{2}(a) \exp \left(\sqrt{2 / a} x_{2}\right)-1}{\beta_{2}(a) \exp \left(\sqrt{2 / a} x_{2}\right)+1}, \quad \text { if } x_{2} \in \mathbb{R}_{+} .
\end{array}\right.
$$


The constants $\beta_{1}(a)$ and $\beta_{2}(a)$ are given explicitly:

$$
\beta_{1}(a)=\frac{\alpha(1+\alpha \sqrt{a})}{\alpha-\sqrt{a}}, \quad \beta_{2}(a)=-\alpha^{2} \beta_{1}(a), \quad \alpha=\frac{1+\sqrt{a}-\sqrt{2(1+a)}}{1-\sqrt{a}} .
$$

Furthermore, we have the following properties:

$$
\left\{\begin{array}{l}
\forall a \in] 0,1\left[, \quad \beta_{1}(a)>1 \text { and } \beta_{2}(a)<-1\right. \\
\forall a \in] 1, \infty\left[, \quad \beta_{1}(a)<-1 \text { and } \beta_{2}(a)>1\right.
\end{array}\right.
$$

and

$$
U^{\prime}(0)=\gamma(a) U(0), \quad \gamma(a)=\alpha \frac{a \alpha^{3}+\sqrt{a} \alpha^{2}+a \alpha+\sqrt{a}}{\alpha^{3}+(4-\sqrt{a}) \alpha^{2}-3 \sqrt{a} \alpha+a} .
$$

As in [18], Theorem 1.5, we get that the solution given by (2.4) is unique in a certain class of functions.

Theorem 2.2. Let $a \in \mathbb{R}_{+} \backslash\{1\}$. Equation (2.3) admits, in the class of functions $\mathcal{C}=\left\{u \in H_{\text {loc }}^{2}\left(\mathbb{R}^{2}\right) \cap\right.$ $L^{\infty}\left(\mathbb{R}^{2}\right): u \geq 0$ in $\left.\mathbb{R}^{2}\right\}$, a unique non-trivial solution given by $(2.4)$.

Proof. Since the proof is very close to that of [18], Theorem 1.5, we sketch only the main steps.

By adjusting the proof of [18], Lemma 4.2 , we obtain that if $u \neq \equiv 0$ solves (2.3), then $0<u<1$ in $\mathbb{R}^{2}$. This permits us, when following step by step the proof of [18], Lemma 4.3 and [24], Lemma 5.3, to get a positive constant $C \in] 0,1[$ such that for any solution $u$ of $(2.3)$ in $\mathcal{C}$, we have

$$
\inf _{x \in \mathbb{R}^{2}} u(x)>C .
$$

Also, we prove in [18], Lemma 4.4 that, for $u \in \mathcal{C}$ a solution of (2.3),

$$
\lim _{x_{2} \rightarrow-\infty}\left(\sup _{x_{1} \in \mathbb{R}}\left(1-u\left(x_{1}, x_{2}\right)\right)\right)=0, \quad \lim _{x_{2} \rightarrow+\infty}\left(\sup _{x_{1} \in \mathbb{R}}\left(\sqrt{a}-u\left(x_{1}, x_{2}\right)\right)\right)=0 .
$$

Now, let $u_{1}, u_{2} \in \mathcal{C}$ be solutions of (2.3). We introduce

$$
\lambda_{*}=\sup \left\{\lambda \in \left[0,1\left[: u_{2}(x)>\lambda u_{1}(x)\right\} .\right.\right.
$$

Then, by (2.9), $\lambda_{*}>0$. We claim that $\lambda_{*}=1$. Once this is shown to hold, Theorem 2.2 is proved.

We argue by contradiction: If $\lambda_{*}<1$, then

$$
\inf _{x \in \mathbb{R}^{2}} w(x)=0,
$$

where $w(x)=u_{2}(x)-\lambda_{*} u_{1}(x)$. Now, let $\left(x_{n}\right)=\left(\left(x_{n}^{1}, x_{n}^{2}\right)\right)$ be a minimizing sequence:

$$
\lim _{n \rightarrow+\infty} w\left(x_{n}\right)=0 .
$$

Since the maximum principle yields that $w(x)>0$ for all $x$, the sequence $\left(x_{n}\right)$ should be unbounded, hence we assume that $\lim _{n \rightarrow+\infty}\left|x_{n}\right|=+\infty$. Also, by (2.10), $\left(x_{n}^{2}\right)$ should be bounded, hence we assume that $\lim _{n \rightarrow+\infty} x_{n}^{2}=b$.

Now, the functions $u_{j}^{n}\left(x_{1}, x_{2}\right)=u_{j}\left(x_{1}+x_{1}^{n}, x_{2}\right), j=1,2$, solve (2.3) in $\mathcal{C}$, and up to extraction of a subsequence, they converge locally to functions $\widetilde{u}_{j} \in C_{\mathrm{loc}}^{2}\left(\overline{\mathbb{R} \times \mathbb{R}_{ \pm}} ; \mathbb{R}\right), j=1,2$. Now, $\widetilde{u}_{1}, \widetilde{u}_{2}$ solve $(2.3)$ in $\mathcal{C}, \widetilde{u}_{2} \geq \lambda_{*} \widetilde{u}_{1}$ and $\widetilde{u}_{2}(0, b)=\lambda_{*} \widetilde{u}_{1}(0, b)$. On the other hand, the strong maximum principle insures that $\widetilde{u}_{2}(x)>\lambda_{*} \widetilde{u}_{1}(x)$ for all $x \in \mathbb{R}^{2}$, hence we have a contradiction. 
Remark 2.3. It is known (see the remark p. 163 in [24]) that when $a=1$, the trivial solution $U \equiv 1$ is the unique positive and bounded solution of equation (2.3).

By a blow-up argument, Theorem 2.2 permits us to obtain the asymptotic behaviour of the minimizer $u_{\varepsilon}$ of (2.1).

Theorem 2.4. Let $a \in \mathbb{R}_{+} \backslash\{1\}$ and $u_{\varepsilon}$ be the positive minimizer of (2.1) introduced in Theorem 2.1. Then, the following asymptotics hold as $\varepsilon \rightarrow 0$ :

$$
\begin{gathered}
\lim _{\varepsilon \rightarrow 0}\left\|u_{\varepsilon}(x)-U\left(\frac{|x|-R}{\varepsilon}\right)\right\|_{L^{\infty}(\Omega)}=0, \\
\forall C>0, \quad \lim _{\varepsilon \rightarrow 0} \varepsilon\left\|u_{\varepsilon}(x)-U\left(\frac{|x|-R}{\varepsilon}\right)\right\|_{W^{1, \infty}\left(\left\{x \in \mathbb{R}^{2}:|R-| x|| \leq C \varepsilon\right\}\right)}=0,
\end{gathered}
$$

where $U$ is the function introduced in (2.5).

In particular, Theorem 2.4 provides a stronger version of Theorem 1.2 when $H=0$.

Proof of Theorem 2.4. Let $(r, \theta)$ be polar coordinates, $0<r<1,-\pi \leq \theta<\pi$, and set

$$
t=r-R, \quad s=R \theta .
$$

Given $s_{0} \in[-R \pi, R \pi[$, we define the rescaled function,

$$
\widetilde{u}_{\varepsilon}(s, t)=u_{\varepsilon}\left((R+\varepsilon t) \mathrm{e}^{\mathrm{i} \varepsilon\left(s-s_{0}\right) / R}\right), \quad \frac{R-1}{\varepsilon}<t<\frac{1-R}{\varepsilon}, \quad-\pi \frac{R}{\varepsilon}<s-s_{0}<\pi \frac{R}{\varepsilon} .
$$

The equation of $\widetilde{u}_{\varepsilon}$ becomes:

$$
\left\{\begin{array}{l}
-\Delta_{\varepsilon} \widetilde{u}_{\varepsilon}=\left(1-\widetilde{u}_{\varepsilon}^{2}\right) \widetilde{u}_{\varepsilon}, \quad \frac{R-1}{\varepsilon}<t<0,\left|s-s_{0}\right|<\pi \frac{R}{\varepsilon}, \\
-\Delta_{\varepsilon} \widetilde{u}_{\varepsilon}=\left(a-\widetilde{u}_{\varepsilon}^{2}\right) \widetilde{u}_{\varepsilon}, \quad 0<t<\frac{1-R}{\varepsilon},\left|s-s_{0}\right|<\pi \frac{R}{\varepsilon}, \\
\frac{\partial \widetilde{u}_{\varepsilon}}{\partial t}\left(\cdot, 0_{-}\right)=\frac{\partial \widetilde{u}_{\varepsilon}}{\partial t}\left(\cdot, 0_{+}\right), \quad \widetilde{u}_{\varepsilon}\left(\cdot, 0_{-}\right)=\widetilde{u}\left(\cdot, 0_{+}\right),
\end{array}\right.
$$

where

$$
\Delta_{\varepsilon}=\left(1-\varepsilon \frac{t}{R}\right)^{-2} \partial_{s}^{2}+\partial_{t}^{2}-\frac{\varepsilon}{(R-\varepsilon t)} \partial_{t} .
$$

Now, by elliptic estimates, the function $\widetilde{u}_{\varepsilon}$ converges to a function $u$ in $W_{\text {loc }}^{2, \infty}\left(\mathbb{R}^{2}\right)$. Furthermore, $u$ solves (2.3) in $\mathcal{C}$, and by [18], Lemma 5.2, there exist constants $k_{0}, c_{0}>0$ such that $u\left(0, k_{0}\right)>c_{0}$. Thus, we conclude by Theorem 2.2 that $u(s, t)=U(t)$, where $U$ is given in (2.4), and therefore, by coming back to the initial scale,

$$
\forall C>0, \quad \forall k \in\{0,1,2\}, \quad \lim _{\varepsilon \rightarrow 0} \varepsilon^{k}\left\|u_{\varepsilon}(s, t)-U\left(\frac{t}{\varepsilon}\right)\right\|_{W^{k, \infty}\left(\left\{\left|s-s_{0}\right| \leq C \varepsilon,|t| \leq C \varepsilon\right\}\right)}=0 .
$$

To prove (2.13), let $x_{\varepsilon}=\left(R+t\left(x_{\varepsilon}\right)\right) \mathrm{e}^{\mathrm{i} s\left(x_{\varepsilon}\right) / R} \in \bar{\Omega}$ such that

$$
\left|u_{\varepsilon}\left(x_{\varepsilon}\right)-U\left(\frac{\left|x_{\varepsilon}\right|-R}{\varepsilon}\right)\right|=\left\|u_{\varepsilon}(x)-U\left(\frac{|x|-R}{\varepsilon}\right)\right\|_{L^{\infty}(\Omega)} .
$$

If $|R-| x_{\varepsilon}|| / \varepsilon$ is bounded, then (2.13) becomes a consequence of (2.15) upon taking $s_{0}=s\left(x_{\varepsilon}\right)$. Otherwise, if $\lim _{\varepsilon \rightarrow 0} \frac{R-\left|x_{\varepsilon}\right|}{\varepsilon}= \pm \infty$, we get again by a blow-up argument that $u_{\varepsilon}\left(x_{\varepsilon}\right) \rightarrow 1$ if the limit is $+\infty$, and $u_{\varepsilon}\left(x_{\varepsilon}\right) \rightarrow \sqrt{a}$ if the limit is $-\infty$. This establishes (2.13) in this case. 
The asymptotic limit (2.14) is also a simple consequence of (2.15). We take $y_{\varepsilon}=\left(R+t\left(y_{\varepsilon}\right)\right) \mathrm{e}^{\mathrm{i} s\left(y_{\varepsilon}\right) / R}$ such that

$$
\left\|\nabla\left(u_{\varepsilon}(x)-U\left(\frac{|x|-R}{\varepsilon}\right)\right)\right\|_{\left.L^{\infty}(|R-| x|| \leq C \varepsilon\}\right)}=\left|\nabla\left(u_{\varepsilon}\left(y_{\varepsilon}\right)-U\left(\frac{\left|y_{\varepsilon}\right|-R}{\varepsilon}\right)\right)\right| .
$$

Then we apply (2.15) with $s_{0}=s\left(y_{\varepsilon}\right)$.

We state also some estimates, taken from [18], Proposition 5.1, that describe the decay of $u_{\varepsilon}$ away from the boundary of $S_{1}$.

Lemma 2.5. Let $k \in \mathbb{N}$. There exist positive constants $\varepsilon_{0}, \delta$ and $C$ such that, for all $\left.\left.\varepsilon \in\right] 0, \varepsilon_{0}\right]$,

$$
\left\|\left(1-u_{\varepsilon}\right) \exp \left(\frac{\delta|R-| x||}{\varepsilon}\right)\right\|_{H^{k}\left(S_{1}\right)}+\left\|\left(\sqrt{a}-u_{\varepsilon}\right) \exp \left(\frac{\delta|R-| x||}{\varepsilon}\right)\right\|_{H^{k}\left(S_{2}\right)} \leq \frac{C}{\varepsilon^{k}} .
$$

Another property that we need is the monotonicity of the function $u_{\varepsilon}$ (recall that, in the setting of Thm. 2.4, $u_{\varepsilon}$ is radial).

Lemma 2.6. With the choice of $\Omega, S_{1}$ and $S_{2}$ as in (1.4), the function $u_{\varepsilon}$ is increasing if $a>1$ and decreasing if $a<1$.

Proof. We only prove the result of the lemma when $a<1$.

Step 1. $u_{\varepsilon}^{\prime}(R) \neq 0$.

Notice that $u_{\varepsilon}$ is positive and satisfies the equations:

$$
\begin{aligned}
& \left.-u_{\varepsilon}^{\prime \prime}-\frac{1}{r} u_{\varepsilon}^{\prime}=\frac{1}{\varepsilon^{2}}\left(1-u_{\varepsilon}^{2}\right) u_{\varepsilon} \quad \text { in }\right] 0, R[ \\
& \left.-u_{\varepsilon}^{\prime \prime}-\frac{1}{r} u_{\varepsilon}^{\prime}=\frac{1}{\varepsilon^{2}}\left(a-u_{\varepsilon}^{2}\right) u_{\varepsilon} \quad \text { in }\right] R, 1[ \\
& u_{\varepsilon}^{\prime}(0)=0, \quad u_{\varepsilon}^{\prime}(1)=0 .
\end{aligned}
$$

Therefore, if $u_{\varepsilon}^{\prime}(R)=0$, then

$$
u_{\varepsilon} \equiv 1 \quad \text { in } S_{1}, \quad u_{\varepsilon} \equiv \sqrt{a} \quad \text { in } S_{2} .
$$

This is impossible since the function $u_{\varepsilon}$ is in $H^{1}(\Omega)$.

Step 2. The function $u_{\varepsilon}$ is decreasing in $[0, R]$.

Recall that, by Theorem 2.1, $\sqrt{a}<u_{\varepsilon}<1$ in $\bar{\Omega}$. It is then easy to verify from equations (2.17) and (2.19) that $u_{\varepsilon}^{\prime \prime}(0)>0$. Let us denote by $\widetilde{u}_{\varepsilon}$ the even extension of $u_{\varepsilon}$ in $]-R, 0[$. Then it is easy to verify that (i) $\widetilde{u}_{\varepsilon} \in C^{2}([-R, R])$; (ii) if $\left.r_{0} \in\right]-R, R\left[\right.$ is a critical point of $\widetilde{u}_{\varepsilon}$, then $\widetilde{u}_{\varepsilon}^{\prime \prime}\left(r_{0}\right)<0$. This shows that every critical point of $\widetilde{u}_{\varepsilon}$ is a local maximum. Consequently, $\widetilde{u}_{\varepsilon}$ should have a unique critical point in $]-R, R[$ and $\widetilde{u}_{\varepsilon}^{\prime}$ should change its sign only at this critical point. Since $\widetilde{u}_{\varepsilon}^{\prime}(0)=0$ and $\widetilde{u}_{\varepsilon}^{\prime \prime}(0)<0$, we deduce that $\widetilde{u}_{\varepsilon}^{\prime}<0$ in $] 0, R\left[\right.$. Therefore, $u_{\varepsilon}$ is decreasing in $[0, R]$.

Step 3. The function $u_{\varepsilon}$ is decreasing in $[R, 1]$.

Notice that from equation (2.18), any critical point $\left.r_{0} \in\right] R, 1\left[\right.$ of $u_{\varepsilon}$ is a local minimum. Thanks to Steps 1 and 2, we have also that $u_{\varepsilon}^{\prime}(R)<0$ and $u_{\varepsilon}^{\prime \prime}(R)>0$.

Let us define the following function

$$
f_{\varepsilon}(r)= \begin{cases}\frac{u_{\varepsilon}^{\prime \prime}(R)}{2}(r-R)^{2}+u_{\varepsilon}^{\prime}(R)(r-R)+u_{\varepsilon}(R), & \text { if } 0<r<R \\ u_{\varepsilon}(r), & \text { if } R \leq r \leq 1, \\ f_{\varepsilon}(2-r), & \text { if } 1<r \leq 2 .\end{cases}
$$


It is clear that $f_{\varepsilon} \in C^{2}([0,2])$ and that it satisfies the following properties: (i) $r_{0}=1$ is a local minimum of $f_{\varepsilon}$; (ii) if $\left.r_{0} \in\right] 0,2$ [is a critical point of $f_{\varepsilon}$, then $r_{0}$ is a local minimum. This proves that $r_{0}=1$ is the only critical point of $f_{\varepsilon}$ in $[0,2]$, and $f_{\varepsilon}^{\prime}$ has a constant sign in $\left[0,1\left[\right.\right.$. Since $u_{\varepsilon}^{\prime}(R)<0$, we deduce that $u_{\varepsilon}^{\prime}<0$ in $] R, 1[$, hence the function $u_{\varepsilon}$ is decreasing.

Finally, we mention without proof that the energy $C_{0}(\varepsilon)(c f .(2.2))$ has the order of $\varepsilon^{-1}$, and we refer to the methods in [18], Section 6, which permit to obtain the leading order asymptotic expansion

$$
C_{0}(\varepsilon)=\frac{c_{1}(a)}{\varepsilon}+c_{2}(a, R)+o(1), \quad(\varepsilon \rightarrow 0),
$$

where $c_{1}(a)$ and $c_{2}(a, R)$ are positive explicit constants.

\subsection{The case with magnetic field}

This section is devoted to a preliminary analysis of the minimizers of (1.1) when $H \neq 0$. The main point that we shall show is how to extract the singular term $C_{0}(\varepsilon)(c f .(2.2))$ from the energy of a minimizer.

Notice that the existence of minimizers is standard starting from a minimizing sequence ( $c f$. e.g. [14]). A standard choice of gauge permits one to assume that the magnetic potential satisfies

$$
\operatorname{div} A=0 \quad \text { in } \Omega, \quad n \cdot A=0 \quad \text { on } \partial \Omega,
$$

where $n$ is the outward unit normal vector of $\partial \Omega$.

With this choice of gauge, one is able to prove (since the boundaries of $\Omega$ and $S_{1}$ are smooth) that a minimizer $(\psi, A)$ is in $C^{1}(\bar{\Omega} ; \mathbb{C}) \times C^{1}\left(\bar{\Omega} ; \mathbb{R}^{2}\right)$. One has also the following regularity (cf. [18], Appendix A),

$$
\psi \in C^{2}\left(\bar{S}_{1} ; \mathbb{C}\right) \cup C^{2}\left(\bar{S}_{2} ; \mathbb{C}\right), \quad A \in C^{2}\left(\bar{S}_{1} ; \mathbb{R}^{2}\right) \cup C^{2}\left(\bar{S}_{2} ; \mathbb{R}^{2}\right) .
$$

The next lemma is inspired from the work of Lassoued and Mironescu (cf. [23]).

Lemma 2.7. Let $(\psi, A)$ be a minimizer of (1.1). Then $0 \leq|\psi| \leq u_{\varepsilon}$ in $\Omega$, where $u_{\varepsilon}$ is the positive minimizer of $(2.1)$.

Moreover, putting $\varphi=\frac{\psi}{u_{\varepsilon}}$, then the energy functional (1.1) splits in the form:

$$
\mathcal{G}_{\varepsilon, H}(\psi, A)=C_{0}(\varepsilon)+\mathcal{F}_{\varepsilon, H}(\varphi, A),
$$

where $C_{0}(\varepsilon)$ has been introduced in $(2.2)$ and the new functional $\mathcal{F}_{\varepsilon, H}$ is defined by:

$$
\mathcal{F}_{\varepsilon, H}(\varphi, A)=\int_{\Omega}\left(u_{\varepsilon}^{2}|(\nabla-i A) \varphi|^{2}+\frac{1}{2 \varepsilon^{2}} u_{\varepsilon}^{4}\left(1-|\varphi|^{2}\right)^{2}+|\operatorname{curl} A-H|^{2}\right) \mathrm{d} x .
$$

Proof. The equality (2.21) results from a direct but some how long calculation, which permits to deduce in particular that $\varphi$ is a solution of the equation

$$
-(\nabla-i A) u_{\varepsilon}^{2}(\nabla-i A) \varphi=\frac{u_{\varepsilon}^{4}}{\varepsilon^{2}}\left(1-|\varphi|^{2}\right)^{2} \varphi .
$$

Proof of $|\psi| \leq u_{\varepsilon}$. It is sufficient to prove that $|\varphi| \leq 1$. We shall invoke an energy argument which we take from [12].

Let us introduce the set

$$
\Omega_{+}=\{x \in \bar{\Omega}:|\varphi(x)|>1\}
$$


together with the functions (defined in $\Omega_{+}$):

$$
f=\frac{\varphi}{|\varphi|}, \quad \widetilde{\varphi}=[|\varphi|-1]_{+} f .
$$

Then, it results from a direct calculation together with the weak-formulation of the equation satisfied by $\varphi$ that

$$
\left.0=\int_{\Omega_{+}}\left(|\nabla| \varphi||^{2}+(|\varphi|-1)|\varphi||(\nabla-i A) f|^{2}+\frac{1}{2 \varepsilon^{2}}(1+|\varphi|)(1-|\varphi|)^{2}|\varphi|\right) u_{\varepsilon}^{2}\right) u_{\varepsilon}^{2} \mathrm{~d} x .
$$

Therefore, this yields that $\Omega_{+}$has measure 0 .

The estimate of the next lemma is very useful for exhibiting a vortex-less regime for minimizers of (1.1). It is due to Béthuel and Rivière [7], but a proof may be found also in [29], Corollary 3.1 (see also [22], Lem. 3.6).

Lemma 2.8. Let $(\psi, A)$ be a minimizer of (1.1). There exist constants $C>0$ and $\left.\left.\varepsilon_{0} \in\right] 0,1\right]$ such that, if the applied magnetic field satisfies $H \ll \frac{1}{\varepsilon}$, then we have

$$
\left.\left.|(\nabla-i A) \psi| \leq \frac{C}{\varepsilon}, \quad \forall \varepsilon \in\right] 0, \varepsilon_{0}\right] .
$$

Now, Lemma 2.8 permits to conclude the following result.

Lemma 2.9. Assume that $(\psi, A)$ is a minimizer of (1.1) and let $\varphi=\frac{\psi}{u_{\varepsilon}}$. There exists a constant $\mu_{0}>0$ such that if

$$
\frac{1}{\varepsilon^{2}} \int_{\Omega}\left(1-|\varphi|^{2}\right)^{2} \mathrm{~d} x \leq \mu_{0}
$$

then $|\varphi| \geq \frac{1}{2}$ in $\bar{\Omega}$.

Proof. Lemma 2.8 and the diamagnetic inequality together yield that

$$
|\nabla| \psi|| \leq|(\nabla-i A) \psi| \leq \frac{C}{\varepsilon}, \quad \text { in } \Omega .
$$

Now, since

we deduce that

$$
\left|\nabla u_{\varepsilon}\right| \leq \frac{C}{\varepsilon}
$$

$$
|\nabla| \varphi|| \leq \frac{C}{\varepsilon} \quad \text { in } \bar{\Omega}
$$

Thus, the result of the lemma becomes a consequence of [8], Theorem III.3.

\section{Analysis of the Meissner state}

Let us recall the definition of $u_{\varepsilon}$ and $C_{0}(\varepsilon)$ in Theorem 2.1 and (2.2) respectively. This section is devoted to the analysis of the following variational problem (3.1):

$$
M_{0}(\varepsilon, H)=\min _{A \in H^{1}\left(\Omega ; \mathbb{R}^{2}\right)} \mathcal{G}_{\varepsilon, H}\left(u_{\varepsilon}, A\right)
$$

Since the function $u_{\varepsilon}$ is real-valued, one gets, for any vector field $A$, the following decomposition:

$$
\mathcal{G}_{\varepsilon, H}\left(u_{\varepsilon}, A\right)=C_{0}(\varepsilon)+\int_{\Omega}\left(\left|A u_{\varepsilon}\right|^{2}+|\operatorname{curl} A-H|^{2}\right) \mathrm{d} x .
$$


Putting further

$$
\begin{gathered}
A=H \mathcal{A} \\
J_{0}(\varepsilon)=\inf _{\mathcal{A} \in H^{1}\left(\Omega ; \mathbb{R}^{2}\right)}\left[\int_{\Omega}\left(\left|\mathcal{A} u_{\varepsilon}\right|^{2}+|\operatorname{curl} \mathcal{A}-1|^{2}\right) \mathrm{d} x\right],
\end{gathered}
$$

we get that

$$
M_{0}(\varepsilon, H)=\inf _{A \in H^{1}\left(\Omega ; \mathbb{R}^{2}\right)} \mathcal{G}_{\varepsilon, H}\left(u_{\varepsilon}, A\right)=C_{0}(\varepsilon)+H^{2} J_{0}(\varepsilon)
$$

and we are reduced to the analysis of the variational problem (3.2).

Starting from a minimizing sequence $(c f .[29])$, it is standard to prove that a minimizer $A_{\varepsilon}$ of $(3.2)$ exists and satisfies the Coulomb gauge condition:

$$
\operatorname{div} A_{\varepsilon}=0 \quad \text { in } \Omega, \quad n \cdot A_{\varepsilon}=0 \quad \text { on } \partial \Omega,
$$

where $n$ is the unit outward normal vector of the boundary of $\Omega$.

Notice also that $A_{\varepsilon}$ satisfies the Euler-Lagrange equations:

$$
\nabla^{\perp} \operatorname{curl} A_{\varepsilon}=u_{\varepsilon}^{2} A_{\varepsilon} \quad \text { in } \Omega, \quad \operatorname{curl} A_{\varepsilon}=1 \quad \text { on } \partial \Omega .
$$

Here $\nabla^{\perp}=\left(-\partial_{x_{2}}, \partial_{x_{1}}\right)$ is the Hodge gradient.

Putting $h_{\varepsilon}=\operatorname{curl} A_{\varepsilon}$, we get from the first equation in (3.3) that $A_{\varepsilon}=\frac{1}{u_{\varepsilon}^{2}} \nabla^{\perp} h_{\varepsilon}$. We get also that $h_{\varepsilon}$ satisfies the equation:

$$
-\operatorname{div}\left(\frac{1}{u_{\varepsilon}^{2}} \nabla h_{\varepsilon}\right)+h_{\varepsilon}=0 \quad \text { in } \Omega, \quad h_{\varepsilon}=1 \quad \text { on } \partial \Omega .
$$

Lemma 3.1. The function $h_{\varepsilon}$ satisfies $0<h_{\varepsilon}<1$ in $\Omega$, and it is the only function solving (3.4).

Moreover, given $\left.R^{\prime} \in\right] 0, R\left[\right.$, there exists a constant $\left.c_{0} \in\right] 0,1\left[\right.$, and for each $a \in \mathbb{R}_{+} \backslash\{1\}$, there exists a positive constant $\varepsilon_{0}<1$ such that,

$$
\left.\left.c_{0} \leq\left|h_{\varepsilon}(x)-1\right|<1, \quad \forall x \in D\left(0, R^{\prime}\right), \forall a \in \mathbb{R}_{+} \backslash\{1\}, \forall \varepsilon \in\right] 0, \varepsilon_{0}\right]
$$

Proof. The property that $0<h_{\varepsilon}<1$ and the uniqueness of $h_{\varepsilon}$ are direct applications of the Strong Maximum Principle.

Let us now prove (3.5). Let us take a set $K \subset S_{1}$ (independent of $\varepsilon$ ). Due to the asymptotic behaviour of $u_{\varepsilon}$ (it remains exponentially close to 1 in $K$, see Lem. 2.5), it results from (3.4) that $h_{\varepsilon}$ is bounded in the $C^{2}$-norm of $K$. Thus, one can extract a subsequence of $h_{\varepsilon}$, still denoted by $h_{\varepsilon}$, that converges to a function $h \in C^{2}(K)$. The function $h$ satisfies the limiting equation,

$$
-\Delta h+h=0 \quad \text { in } K
$$

By the Strong Maximum Principle, $0<h<1$ in $K$. Let $h_{0}$ be the solution of the equation

$$
-\Delta h_{0}+h_{0}=0 \quad \text { in } K, \quad h_{0}=1 \quad \text { on } \partial K
$$

Then, by the Strong Maximum Principle, $0<h \leq h_{0}<1$ in $K$. This achieves the proof of the lemma. 
Lemma 3.2. With the assumption (1.4), the function $h_{\varepsilon}$ is radial, i.e. $h_{\varepsilon}(x)=\widetilde{h}_{\varepsilon}(|x|)$, with $\widetilde{h}_{\varepsilon}$ being an increasing function.

Proof. That $h_{\varepsilon}$ is radial follows by the uniqueness of the solution of (3.4) and by the fact that $u_{\varepsilon}$ is also radial.

The solution $h_{\varepsilon}$ being radial, i.e.

$$
h_{\varepsilon}(x)=\widetilde{h}_{\varepsilon}(|x|), \quad \forall x \in \Omega,
$$

let us show that the function $\widetilde{h}_{\varepsilon}$ is increasing. For simplicity of notation, we shall remove the tilde and write $h_{\varepsilon}$ for $\widetilde{h}_{\varepsilon}$. Notice that $h_{\varepsilon}$ satisfies the differential equation:

$$
\left\{\begin{array}{l}
-h_{\varepsilon}^{\prime \prime}(r)-\frac{1}{r} h_{\varepsilon}^{\prime}(r)+2 \frac{u_{\varepsilon}^{\prime}(r)}{u_{\varepsilon}(r)} h_{\varepsilon}^{\prime}(r)+u_{\varepsilon}^{2}(r) h_{\varepsilon}(r)=0, \quad 0<r<1 \\
h_{\varepsilon}^{\prime}(0)=0, \quad h_{\varepsilon}(1)=1
\end{array}\right.
$$

Let us calculate $h_{\varepsilon}^{\prime \prime}(0)$. Since $h_{\varepsilon}^{\prime}(0)=0$, we have $h_{\varepsilon}^{\prime \prime}(0)=\lim _{r \rightarrow 0} \frac{h_{\varepsilon}^{\prime}(r)}{r}$. Substituting in (3.6), we get that

$$
h_{\varepsilon}^{\prime \prime}(0)=\frac{1}{2} u_{\varepsilon}^{2}(0) h_{\varepsilon}(0)>0
$$

Let us introduce the even extension of $h_{\varepsilon}$, namely the function

$$
f_{\varepsilon}(r)=\left\{\begin{array}{lc}
h_{\varepsilon}(r) & (r>0) \\
h_{\varepsilon}(-r) & (r<0)
\end{array}\right.
$$

Then $f_{\varepsilon}$ satisfies the equation,

$$
\left.-f_{\varepsilon}^{\prime \prime}(r)-\frac{1}{|r|} f_{\varepsilon}^{\prime}(r)+2 \frac{\widetilde{u}_{\varepsilon}^{\prime}(r)}{\widetilde{u}_{\varepsilon}(r)} f_{\varepsilon}^{\prime}(r)+\widetilde{u}_{\varepsilon}^{2}(r) f_{\varepsilon}(r)=0, \quad r \in\right]-r_{2}, r_{2}[\backslash\{0\},
$$

and it attains a local minimum at 0 . We emphasize also here that $\widetilde{u}_{\varepsilon}$ denotes the even extension of $u_{\varepsilon}$.

If $\left.r_{0} \in\right]-1,1\left[\right.$ (with $r_{0} \neq 0$ ) is a critical point of $f_{\varepsilon}$, then it follows from (3.8) that:

$$
f_{\varepsilon}^{\prime \prime}\left(r_{0}\right)=\widetilde{u}_{\varepsilon}^{2}\left(r_{0}\right) f_{\varepsilon}\left(r_{0}\right)>0 .
$$

If $r_{0}=0$, the conclusion $f_{\varepsilon}^{\prime \prime}(0)>0$ still holds, thanks to (3.7).

Now these observations lead to the conclusion that $f_{\varepsilon}$ attains its minimum at a unique point, and that this point is the only critical point for $f_{\varepsilon}$. As we know that $f_{\varepsilon}^{\prime}(0)=0$, we get that $f_{\varepsilon}$ attains its minimum at 0 and that it is increasing in $[0,1[$. This achieves the proof of the lemma.

As we shall see, the next lemma will play a distinguished role in the control of the minimizing energy of 'vortex balls'.

Lemma 3.3. The following estimate holds

$$
\left.\left.\left\|\frac{1}{u_{\varepsilon}^{2}} \nabla h_{\varepsilon}\right\|_{L^{\infty}(\Omega)} \leq 1, \quad \forall \varepsilon \in\right] 0,1\right] \quad \forall a \in \mathbb{R}_{+} \backslash\{1\}
$$

Proof. Notice that by Lemma $3.2, h_{\varepsilon}$ is radial. Then the equation for $h_{\varepsilon}$ can be written in the form:

$$
\left.-\left(\frac{h_{\varepsilon}^{\prime}}{u_{\varepsilon}^{2}}\right)^{\prime}(r)-\frac{1}{r} \frac{h_{\varepsilon}^{\prime}}{u_{\varepsilon}^{2}}(r)+h_{\varepsilon}(r)=0, \quad \forall r \in\right] 0,1[.
$$


Integrating this equation between 0 and $r \in] 0,1\left[\right.$ and using the fact that $h_{\varepsilon}$ is increasing, $h_{\varepsilon}^{\prime} \geq 0$, we obtain:

$$
\left(\frac{h_{\varepsilon}^{\prime}}{u_{\varepsilon}^{2}}\right)(r) \leq \int_{0}^{r} h_{\varepsilon}(\widetilde{r}) \mathrm{d} \widetilde{r} \leq r\left\|h_{\varepsilon}\right\|_{L^{\infty}([0,1])} \leq 1,
$$

which is the result of the lemma.

Let us introduce the set

$$
\Lambda_{\varepsilon}=\left\{x \in \Omega: \frac{1-h_{\varepsilon}(x)}{u_{\varepsilon}^{2}(x)}=\max _{\bar{\Omega}} \frac{1-h_{\varepsilon}}{u_{\varepsilon}^{2}}\right\} .
$$

Theorem 3.4. The following two assertions hold.

(1) If $a>1$, the function $\frac{1-h_{\varepsilon}}{u_{\varepsilon}^{2}}$ is strictly decreasing, and $\Lambda_{\varepsilon}=\{0\}$.

(2) There exists $\left.a_{0} \in\right] 0,1[$ such that, for all $a \in] 0, a_{0}\left[\right.$, the set $\Lambda_{\varepsilon}$ is a circle $\partial D\left(0, R_{\varepsilon}\right)$ localized strictly in $S_{2}$ as $\varepsilon \rightarrow 0$ in the sense that given $\left.\alpha \in\right] 0,1[$, we have,

$$
\varepsilon \ll R_{\varepsilon}-R \ll \varepsilon^{\alpha}, \quad(\varepsilon \rightarrow 0) .
$$

Moreover, there exists a positive constant $C>0$ such that

$$
\left|\nabla u_{\varepsilon}(x)\right| \leq C, \quad \forall x \in S_{2} \backslash D\left(0, R_{\varepsilon}\right) .
$$

Proof. The proof of the first assertion is straightforward: When $a>1$, the functions $u_{\varepsilon}$ and $h_{\varepsilon}$ are strictly increasing, hence

$$
\left(\frac{1-h_{\varepsilon}}{u_{\varepsilon}^{2}}\right)^{\prime}=-\frac{u_{\varepsilon} h_{\varepsilon}^{\prime}+2\left(1-h_{\varepsilon}\right) u_{\varepsilon}^{\prime}}{u_{\varepsilon}^{3}}<0 .
$$

The proof of the second assertion of the corollary is more delicate. We present it in five steps.

Step. 1. Proof of $\varepsilon \ll R_{\varepsilon}-R$.

Choose $x_{\varepsilon} \in \Lambda_{\varepsilon}$ and let $r_{\varepsilon}=\left|x_{\varepsilon}\right|$. Then $r_{\varepsilon} \in[0,1[$. Thanks to Lemmas 3.1-3.3, we have:

$$
\liminf _{\varepsilon \rightarrow 0} \frac{1-h_{\varepsilon}\left(r_{\varepsilon}\right)}{u_{\varepsilon}^{2}\left(r_{\varepsilon}\right)} \geq c_{0}, \quad \limsup _{\varepsilon \rightarrow 0} r_{\varepsilon}<1
$$

Since $r_{\varepsilon}$ is a critical point of the function $\frac{1-h_{\varepsilon}}{u_{\varepsilon}^{2}}$, we have

$$
u_{\varepsilon}^{\prime}\left(r_{\varepsilon}\right)=\frac{u_{\varepsilon}\left(r_{\varepsilon}\right) h_{\varepsilon}^{\prime}\left(r_{\varepsilon}\right)}{2\left(1-h_{\varepsilon}\left(r_{\varepsilon}\right)\right)} .
$$

Then, by Lemma 3.3, $\left|u_{\varepsilon}^{\prime}\left(r_{\varepsilon}\right)\right| \leq C$ for an explicit constant $C>0$. By Theorem 2.4, we should have

$$
\left|R-r_{\varepsilon}\right| \gg \varepsilon \quad \text { as } \varepsilon \rightarrow 0 .
$$

Assume by contradiction that $r_{\varepsilon}<R$. Then Theorem 2.4 yields that $\lim _{\varepsilon \rightarrow 0} u_{\varepsilon}\left(r_{\varepsilon}\right)=1$.

Let $\alpha \in] 0,1\left[\right.$ and choose $\left.r^{\prime} \in\right] 0, R[$ such that

$$
\left|h_{\varepsilon}\left(R+\varepsilon^{\alpha}\right)-h_{\varepsilon}\left(r^{\prime}\right)\right| \leq \frac{1}{2}\left|1-h_{\varepsilon}\left(r^{\prime}\right)\right| .
$$

This choice of $r^{\prime}$ is always possible, thanks to Lemmas 3.1 and 3.3 . 
Now, notice that, as $\varepsilon \rightarrow 0$,

$$
\begin{aligned}
\frac{1-h_{\varepsilon}\left(R+\varepsilon^{\alpha}\right)}{u_{\varepsilon}^{2}\left(R+\varepsilon^{\alpha}\right)} & \geq \frac{1}{2} \frac{\left|1-h_{\varepsilon}\left(r^{\prime}\right)\right|}{u_{\varepsilon}^{2}\left(R+\varepsilon^{\alpha}\right)} \\
& \geq \frac{c_{0}}{2 u_{\varepsilon}^{2}\left(R+\varepsilon^{\alpha}\right)} \quad\left(c_{0} \in\right] 0,1[\text { given in Lem. 3.1) } \\
& =\frac{c_{0}}{2 a}(1+o(1)) \quad(\text { by Thm. 2.4). }
\end{aligned}
$$

On the other hand, by the definition of $r_{\varepsilon}$,

$$
\frac{1-h_{\varepsilon}\left(R+\varepsilon^{\alpha}\right)}{u_{\varepsilon}^{2}\left(R+\varepsilon^{\alpha}\right)} \leq \frac{1-h_{\varepsilon}\left(r_{\varepsilon}\right)}{u_{\varepsilon}^{2}\left(r_{\varepsilon}\right)},
$$

and since $\lim _{\varepsilon \rightarrow 0} u_{\varepsilon}\left(r_{\varepsilon}\right)=1$, we get

$$
\frac{1-h_{\varepsilon}\left(R+\varepsilon^{\alpha}\right)}{u_{\varepsilon}^{2}\left(R+\varepsilon^{\alpha}\right)} \leq 1+o(1) \quad \text { as } \varepsilon \rightarrow 0 .
$$

Therefore, by choosing $a \in] 0, \frac{c_{0}}{2}[,(3.15)$ leads to a contradiction. By putting

$$
R_{\varepsilon}=\min _{x \in \Lambda_{\varepsilon}}|x|
$$

we get the desired statement: $\varepsilon \ll R_{\varepsilon}-R$ as $\varepsilon \rightarrow 0$.

Step 2. Proof of $R_{\varepsilon}-R \ll 1$.

Assume that there exists $\left.R_{1} \in\right] R, 1\left[\right.$ such that, up to extraction of a subsequence, $R_{\varepsilon} \rightarrow R_{1}$ as $\varepsilon \rightarrow 0$. Let $\delta=\frac{1}{2} \min \left(\left|R-R_{1}\right|, 1\right)$. We may assume, by extracting a subsequence, that $h_{\varepsilon}\left(R_{1}-\delta\right) \rightarrow c_{*}$ for some constant $\left.c_{*} \in\right] 0,1\left[\right.$. Then, by standard elliptic estimates, there exists a function $h_{*} \in C^{2}\left(\overline{D(0,1) \backslash D\left(0, R_{1}-\delta\right)}\right)$ such that, upon the extraction of a subsequence, we have,

$$
h_{\varepsilon} \rightarrow h_{*} \quad \text { in } C^{2}\left(\overline{D(0,1) \backslash D\left(0, R_{1}-\delta\right)}\right)
$$

and $h_{*}$ is a radial function and the unique solution of the equation

$$
\left\{\begin{array}{l}
-\Delta h_{*}+a h_{*}=0 \quad \text { in } D(0,1) \backslash \overline{D\left(0, R_{1}-\delta\right)} \\
h_{*}=c_{*} \quad \text { on } \partial D\left(0, R_{1}-\delta\right), \quad h_{*}=1 \quad \text { on } \partial D(0,1) .
\end{array}\right.
$$

A simple application of the maximum principle yields that $c_{*}<h_{*}<1$ in $D(0,1) \backslash \overline{D\left(0, R_{1}-\delta\right)}$. Therefore, there exists a constant $M>0$ such that

$$
h_{*}\left(R_{1}-\delta\right)<h_{*}\left(R_{1}\right)-M
$$

Consequently, when $\varepsilon$ is sufficiently small, we get the lower bound:

$$
\frac{1-h_{\varepsilon}\left(R_{1}-\delta\right)}{u_{\varepsilon}^{2}\left(R_{1}-\delta\right)}>\frac{1-h_{\varepsilon}\left(R_{1}\right)}{u_{\varepsilon}^{2}\left(R_{1}\right)}+\frac{M}{2},
$$

and the same estimate holds when we replace $R_{1}$ by $R_{\varepsilon}$ and $\frac{M}{2}$ by $\frac{M}{4}$. This contradicts the definition of $R_{\varepsilon}$, proving thus the desired property: $R_{\varepsilon}-R \ll 1$ as $\varepsilon \rightarrow 0$. 
Step 3. Finer localization: Proof of $R_{\varepsilon}-R \ll \varepsilon^{\alpha}$.

Assume that there exists $\alpha \in] 0,1\left[\right.$ such that, up to the extraction of a subsequence, $R_{\varepsilon}>R+\varepsilon^{\alpha}$.

Let $\left.\alpha^{\prime} \in\right] \alpha, 1\left[\right.$ and set $\delta_{\varepsilon}=R_{\varepsilon}-R-\varepsilon^{\alpha^{\prime}}$. Notice that

$$
\delta_{\varepsilon} \geq \frac{\varepsilon^{\alpha}}{2} \quad \text { when } \varepsilon \text { is small enough. }
$$

Thanks to (3.14) and Lemma $2.5, h_{\varepsilon}^{\prime}\left(R_{\varepsilon}\right)$ is exponentially small as $\varepsilon \rightarrow 0$. Thus, from the equations satisfied by $h_{\varepsilon}$, we may assume that up to the extraction of a subsequence,

$$
h_{\varepsilon}^{\prime \prime}\left(R_{\varepsilon}\right) \rightarrow \lambda_{0} \text { as } \varepsilon \rightarrow 0, \quad \lambda_{0}>0 .
$$

Now, applying Taylor's formula to the function $h_{\varepsilon}$, we get

$$
h_{\varepsilon}\left(R_{\varepsilon}-\delta_{\varepsilon}\right)=h_{\varepsilon}\left(R_{\varepsilon}\right)+\lambda_{0} \delta_{\varepsilon}^{2}+o\left(\delta_{\varepsilon}^{2}\right) \quad \text { as } \varepsilon \rightarrow 0 .
$$

Consequently, thanks again to Lemma 2.5, we deduce that

$$
\begin{aligned}
\frac{1-h_{\varepsilon}\left(R_{\varepsilon}-\delta_{\varepsilon}\right)}{u_{\varepsilon}^{2}\left(R_{\varepsilon}-\delta_{\varepsilon}\right)} & =\frac{1-h_{\varepsilon}\left(R_{\varepsilon}\right)}{u_{\varepsilon}^{2}\left(R_{\varepsilon}\right)}-\lambda_{0} \delta_{\varepsilon}^{2}+o\left(\delta_{\varepsilon}^{2}\right) \\
& <\frac{1-h_{\varepsilon}\left(R_{\varepsilon}\right)}{u_{\varepsilon}^{2}\left(R_{\varepsilon}\right)}-\frac{\lambda_{0}}{2} \delta_{\varepsilon}^{2} .
\end{aligned}
$$

Since the function $[0,1] \ni r \mapsto \frac{1-h_{\varepsilon}(r)}{u_{\varepsilon}^{2}(r)}$ achieves its maximum on $R_{\varepsilon}$, we get a contradiction. Therefore, we have proved the desired localization for $R_{\varepsilon}$ : Given $\left.\alpha \in\right] 0,1\left[, R_{\varepsilon}-R \ll \varepsilon^{\alpha}\right.$ as $\varepsilon \rightarrow 0$.

Step 4. Upper Bound for $\left|\nabla u_{\varepsilon}\right|$.

Let us prove now (3.12). We know that $\left|u_{\varepsilon}^{\prime}\left(R_{\varepsilon}\right)\right| \leq C$, for some explicit constant $C>0$. On the other hand, by Lemma 2.6, the function $u_{\varepsilon}$ is decreasing when $a<1$, hence $u_{\varepsilon}^{\prime} \leq 0$ in $\left.] R, 1\right]$. So it is sufficient to prove that $u_{\varepsilon}^{\prime}$ is increasing in $[R, 1]$. Actually, coming back to the equation of $u_{\varepsilon}$, we have, thanks to Theorem 2.1,

$$
\left.u_{\varepsilon}^{\prime \prime}=-\frac{1}{r} u_{\varepsilon}^{\prime}-\frac{1}{\varepsilon^{2}}\left(a-u_{\varepsilon}^{2}\right) u_{\varepsilon}>0 \quad \text { in }\right] R, 1[,
$$

hence we have the desired property regarding the monotonicity of $u_{\varepsilon}^{\prime}$. This achieves the proof of (3.12).

Step 5. The function $[0,1] \ni r \mapsto \frac{1-h_{\varepsilon}(r)}{u_{\varepsilon}^{2}(r)}$ achieves its maximum on a unique point.

Let us prove now that $\Lambda_{\varepsilon}=\partial D\left(0, R_{\varepsilon}\right)$, i.e. the radial function $\frac{1-h_{\varepsilon}}{u_{\varepsilon}^{2}}$ attains its maximum uniquely at $R_{\varepsilon}$.

By Lemma 3.1, there exists a constant $\left.R_{*} \in\right] R, 1\left[\right.$ such that any maximum point $x \in \Lambda_{\varepsilon}$ should satisfy $R<|x|<R_{*}$. Let $\left.r_{\varepsilon} \in\right] R_{\varepsilon}, R_{*}\left[\right.$ be a critical point of $\frac{1-h_{\varepsilon}}{u_{\varepsilon}^{2}}$. Then,

$$
\left(\frac{1-h_{\varepsilon}}{u_{\varepsilon}^{2}}\right)^{\prime \prime}=\frac{-3 f_{\varepsilon}}{u_{\varepsilon}^{4}}
$$

where

$$
f_{\varepsilon}=u_{\varepsilon}^{\prime} h_{\varepsilon}^{\prime}-\frac{1}{r} h_{\varepsilon}^{\prime}+u_{\varepsilon}^{2} h_{\varepsilon}+2\left(1-h_{\varepsilon}\right) u_{\varepsilon}^{\prime \prime} .
$$

It is sufficient to prove that $f_{\varepsilon}\left(r_{\varepsilon}\right)>0$. We distinguish between two cases:

$$
\text { (i) } \limsup _{\varepsilon \rightarrow 0} u_{\varepsilon}^{\prime \prime}\left(r_{\varepsilon}\right)=\infty \text {, or (ii) } \limsup _{\varepsilon \rightarrow 0} u_{\varepsilon}^{\prime \prime}\left(r_{\varepsilon}\right)<\infty \text {. }
$$


In case (i), since $u_{\varepsilon}^{\prime}$ is bounded in $\left[R_{\varepsilon}, 1[\right.$, we deduce easily that as $\varepsilon \rightarrow 0$,

$$
f_{\varepsilon}\left(r_{\varepsilon}\right)>0
$$

In case (ii), it is easy to verify that $u_{\varepsilon}^{\prime \prime}$ is decreasing in $] R_{\varepsilon}, 1[$. Hence there exists a constant $C>0$ such that, up to the extraction of a subsequence, $u_{\varepsilon}^{\prime \prime}(r) \leq C$ in $\left[r_{\varepsilon}, 1[\right.$.

By the mean value theorem, we deduce that

$$
\left|u_{\varepsilon}^{\prime}\left(r_{\varepsilon}\right)-u_{\varepsilon}^{\prime}\left(r_{\varepsilon}+\varepsilon^{\alpha}\right)\right| \leq C \varepsilon^{\alpha} .
$$

Thus, we get by Lemma 2.5 that $\left|u_{\varepsilon}^{\prime}\left(r_{\varepsilon}\right)\right| \ll 1$ as $\varepsilon \rightarrow 0$, and consequently, we get by (3.14) that $h_{\varepsilon}^{\prime}\left(r_{\varepsilon}\right) \ll 1$ as $\varepsilon \rightarrow 0$.

Now, this yields in this case that $f_{\varepsilon}\left(r_{\varepsilon}\right)>0$. Therefore, we have proved all the statements of the theorem.

Let us introduce the function

together with

$$
\Omega \ni x \mapsto \xi_{\varepsilon}(x)=\frac{h_{\varepsilon}(x)-1}{u_{\varepsilon}^{2}(x)},
$$

$$
\lambda_{\varepsilon}=\max _{x \in \bar{\Omega}}\left|\xi_{\varepsilon}(x)\right| .
$$

Corollary 3.5. Let $\left.a_{0} \in\right] 0,1[$ be the constant of Theorem 3.4. For all $a \in] 0, a_{0}[$, there exist positive constants $\delta_{*}$ and $\varepsilon_{0}$ such that:

$$
\left\{\begin{array}{l}
\left.\left.\forall x \in \Omega \quad \text { s.t. }|| x|-R|^{2} \geq|\ln \varepsilon|^{-1 / 2}, \quad \forall \varepsilon \in\right] 0, \varepsilon_{0}\right] \\
\xi_{\varepsilon}(x) \geq-\lambda_{\varepsilon}+\delta_{*}|\ln \varepsilon|^{-1 / 2}
\end{array}\right.
$$

Proof. We make the following claim:

$$
\exists c_{0}>0, \quad \xi_{\varepsilon}(x) \geq-\lambda_{\varepsilon}+c_{0} \quad \text { when }|x| \leq R-|\ln \varepsilon|^{-1 / 4} .
$$

Once we prove (3.18), we deduce the conclusion of the corollary when $|x|<R-|\ln \varepsilon|^{-1 / 4}$.

The proof of (3.18) is rather easy. First, notice that, putting $r_{\varepsilon}=R-\varepsilon^{\alpha}$ with $\left.\alpha \in\right] 0,1[$, we have by Lemma 3.3

$$
h_{\varepsilon}\left(r_{\varepsilon}\right) \geq h_{\varepsilon}\left(R_{\varepsilon}\right)+\mathcal{O}\left(\varepsilon^{\alpha}\right) .
$$

On the other hand, Theorem 3.1 yields that the function $r \mapsto \frac{h_{\varepsilon}(r)-1}{u_{\varepsilon}^{2}(r)}$ is decreasing in $[0, R]$. Thus, for all $r \in\left[0, R-|\ln \varepsilon|^{-1 / 4}\right]$, we have

Therefore,

$$
\frac{h_{\varepsilon}(r)-1}{u_{\varepsilon}^{2}(r)} \geq \frac{h_{\varepsilon}\left(r_{\varepsilon}\right)-1}{u_{\varepsilon}^{2}\left(r_{\varepsilon}\right)} .
$$

Invoking Lemma 2.5, we deduce that

$$
\xi_{\varepsilon}(r) \geq \frac{h_{\varepsilon}\left(R_{\varepsilon}\right)-1}{u_{\varepsilon}^{2}\left(r_{\varepsilon}\right)}+\mathcal{O}\left(\varepsilon^{\alpha}\right)
$$

where $\left.c_{0} \in\right] 0,\left(\frac{1}{a}-1\right) \liminf _{\varepsilon \rightarrow 0}\left(1-h_{\varepsilon}\left(R_{\varepsilon}\right)\right)[$.

$$
\xi_{\varepsilon}(r) \geq-\lambda_{\varepsilon}+c_{0}
$$

Now, let us prove the conclusion of the corollary when $R+|\ln \varepsilon|^{-1 / 4} \leq|x| \leq 1$. By Lemma 2.5, it is sufficient to find $\delta>0$ and $r_{0}>0$ such that

$$
h_{\varepsilon}(r) \geq h_{\varepsilon}\left(R_{\varepsilon}\right)+\delta \max \left(|r-R|^{2},|\ln \varepsilon|^{-1 / 2}\right), \quad R+|\ln \varepsilon|^{-1 / 4} \leq r \leq R+r_{0} .
$$

To prove (3.19), we deal separately with the case whether $\liminf _{\varepsilon \rightarrow 0} h_{\varepsilon}^{\prime}\left(R_{\varepsilon}\right)=0$ or $\liminf _{\varepsilon \rightarrow 0} h_{\varepsilon}^{\prime}\left(R_{\varepsilon}\right)>0$. 
Proof of (3.19) when $\liminf _{\varepsilon \rightarrow 0} h_{\varepsilon}^{\prime}\left(R_{\varepsilon}\right)=0$. In this case, there exists $c_{0}>0$ such that, up to the extraction of a subsequence,

$$
h_{\varepsilon}^{\prime}\left(R_{\varepsilon}\right) \rightarrow 0, \quad h_{\varepsilon}^{\prime \prime}\left(R_{\varepsilon}\right) \rightarrow c_{0} \quad \text { as } \varepsilon \rightarrow 0 .
$$

Set $r_{\varepsilon}=R+\varepsilon^{\alpha}$ where $\left.\alpha \in\right] 0,1[$ is given. By Theorem 3.4, we have

$$
h_{\varepsilon}^{\prime}\left(r_{\varepsilon}\right) \rightarrow 0, \quad h_{\varepsilon}^{\prime \prime}\left(r_{\varepsilon}\right) \rightarrow c_{0} \quad \text { as } \varepsilon \rightarrow 0
$$

Moreover, by Lemma 2.5 and the equation of $h_{\varepsilon}, h_{\varepsilon}^{\prime \prime \prime}(r)$ is bounded in $\left[r_{\varepsilon}, 1\right]$. Therefore, applying Taylor's formula up to the order 2 , we get a positive constant $\left.r_{0} \in\right] 0,1[$ such that

$$
\begin{aligned}
h_{\varepsilon}(r) & =h_{\varepsilon}\left(r_{\varepsilon}\right)+h_{\varepsilon}^{\prime}\left(r_{\varepsilon}\right)\left(r-r_{\varepsilon}\right)+\frac{1}{2} h_{\varepsilon}^{\prime \prime}\left(r_{\varepsilon}\right)\left(r-r_{\varepsilon}\right)^{2}+\mathcal{O}\left(\left|r-r_{\varepsilon}\right|^{3}\right) \\
& \geq h_{\varepsilon}\left(r_{\varepsilon}\right)+\frac{c_{0}}{2}\left(r-r_{\varepsilon}\right)^{2}
\end{aligned}
$$

provided that $0<r-r_{\varepsilon}<r_{0}$.

Thanks to Theorem $3.4, \varepsilon<R_{\varepsilon}-R<\varepsilon^{\alpha}$. Hence by Lemma 3.3,

$$
h_{\varepsilon}\left(r_{\varepsilon}\right)=h_{\varepsilon}\left(R_{\varepsilon}\right)+\mathcal{O}\left(\varepsilon^{\alpha}\right)
$$

Therefore, when $|\ln \varepsilon|^{-1 / 2}<r-R_{\varepsilon}<r_{0}$, (3.20) is nothing but (3.19).

Proof of (3.19) when $\liminf _{\varepsilon \rightarrow 0} h_{\varepsilon}^{\prime}\left(R_{\varepsilon}\right)>0$. We may assume in this case that $h_{\varepsilon}^{\prime}\left(R_{\varepsilon}\right) \rightarrow c_{0}>0$ as $\varepsilon \rightarrow 0$. By Theorem 3.4 and the equation of $h_{\varepsilon}, h_{\varepsilon}^{\prime \prime}(r)$ is bounded in $\left[R_{\varepsilon}, 1\right]$ independently of $\varepsilon$.

We apply again Taylor's formula

$$
\begin{aligned}
h_{\varepsilon}(r) & =h_{\varepsilon}\left(R_{\varepsilon}\right)+h_{\varepsilon}^{\prime}\left(R_{\varepsilon}\right)\left(r-R_{\varepsilon}\right)+\mathcal{O}\left(\left|r-R_{\varepsilon}\right|^{2}\right) \\
& \geq h_{\varepsilon}\left(R_{\varepsilon}\right)+\frac{c_{0}}{2}\left|r-R_{\varepsilon}\right| .
\end{aligned}
$$

This is nothing but again (3.19). This achieves the proof of the corollary.

\section{LOWER BOUND OF THE ENERGY}

\subsection{Vortex-balls}

In this section we construct suitable 'vortex-balls' providing a lower bound of the energy of minimizers of (1.1). Recall the decomposition of the energy in Lemma 2.7, which permits us to work with the 'reduced energy functional' $\mathcal{F}_{\varepsilon, H}$.

Notice that, by using $\left(u_{\varepsilon}, \frac{1}{u_{\varepsilon}^{2}} \nabla^{\perp} h_{\varepsilon}\right)$ as a test configuration for the function (1.1), we deduce the upper bound:

$$
\mathcal{F}_{\varepsilon, H}(\varphi, A) \leq H^{2} J_{0}(\varepsilon)
$$

where $\varphi=\psi / u_{\varepsilon},(\psi, A)$ a minimizer of (1.1), and $J_{0}(\varepsilon)$ is introduced in (3.2),

$$
J_{0}(\varepsilon)=\int_{\Omega}\left(\frac{1}{u_{\varepsilon}^{2}}\left|\nabla h_{\varepsilon}\right|^{2}+\left|h_{\varepsilon}-1\right|^{2}\right) \mathrm{d} x .
$$

We shall always work under the hypothesis that there exists a positive constant $C>0$ such that the applied magnetic field $H$ satisfies

$$
H \leq C|\ln \varepsilon| .
$$

The upper bound (4.1) provides us, as in [29], with the construction of suitable 'vortex-balls'. 
Proposition 4.1. Let $(\psi, A)$ be a minimizer of (1.1) and $\varphi=\frac{\psi}{u_{\varepsilon}}$. Then, under the hypotheses (4.3), for each $p \in] 1,2\left[\right.$, there exist a constant $C>0$ and a finite family of disjoint balls $\left\{B\left(\left(a_{i}, r_{i}\right)\right\}_{i \in I}\right.$ satisfying the following properties:

(1) $w=\left\{x \in \bar{\Omega}:|\varphi(x)| \leq 1-|\ln \varepsilon|^{-4}\right\} \subset \bigcup_{i \in I} B\left(a_{i}, r_{i}\right)$.

(2) $\sum_{i \in I} r_{i} \leq C|\ln \varepsilon|^{-10}$.

(3) Letting $d_{i}$ be the degree of the function $\varphi /|\varphi|$ restricted to $\partial B\left(a_{i}, r_{i}\right)$ if $B\left(a_{i}, r_{i}\right) \subset \Omega$ and $d_{i}=0$ otherwise, then we have:

$$
\begin{aligned}
& \int_{B\left(a_{i}, r_{i}\right) \backslash \omega} u_{\varepsilon}^{2}|(\nabla-i A) \varphi|^{2} \mathrm{~d} x+\int_{B\left(a_{i}, r_{i}\right)}|\operatorname{curl} A-H|^{2} \mathrm{~d} x \geq 2 \pi\left|d_{i}\right|\left(\min _{B\left(a_{i}, r_{i}\right)} u_{\varepsilon}^{2}\right)(|\ln \varepsilon|-C \ln |\ln \varepsilon|) . \\
& \text { (4) }\left\|2 \pi \sum_{i \in I} d_{i} \delta_{a_{i}}-\operatorname{curl}\left(A+\left(i \varphi, \nabla_{A} \varphi\right)\right)\right\|_{W_{0}^{-1, p}(\Omega)} \leq C|\ln \varepsilon|^{-4} .
\end{aligned}
$$

We follow the usual terminology and call the balls constructed in Proposition 4.1 'vortex-balls'. The proof of Proposition 4.1 is very similar to that of [22], Proposition 5.2, and is actually a simple consequence of the analysis of [29].

Proposition 4.1 permits us to prove the following theorem.

Theorem 4.2. Let $(\psi, A)$ be a minimizer of (1.1) and $\varphi=\frac{\psi}{u_{\varepsilon}}$. Then, under the hypothesis (4.3), there exist a constant $C>0$ and a finite family of disjoint balls $\left\{B\left(\left(a_{i}, r_{i}\right)\right\}_{i \in I}\right.$ such that:

(1) $\sum_{i \in I} r_{i} \leq C|\ln \varepsilon|^{-10}$.

(2) $|\varphi| \geq \frac{1}{2}$ on $\Omega \backslash \cup_{i} B\left(a_{i}, r_{i}\right)$.

(3) Letting $d_{i}$ be the degree of the function $\varphi /|\varphi|$ restricted to $\partial B\left(a_{i}, r_{i}\right)$ if $B\left(a_{i}, r_{i}\right) \subset \Omega$ and $d_{i}=0$ otherwise, then we have:

$$
\begin{aligned}
\mathcal{F}_{\varepsilon, H}(\varphi, A) \geq & H^{2} J_{0}(\varepsilon)+\int_{\Omega \backslash \cup_{i} B\left(a_{i}, r_{i}\right)} \frac{1}{u_{\varepsilon}^{2}}\left|\nabla\left(h-H h_{\varepsilon}\right)\right|^{2} \mathrm{~d} x+\int_{\Omega}\left|h-H h_{\varepsilon}\right|^{2} \mathrm{~d} x \\
& +2 \pi \sum_{i \in I}\left[\left(\min _{B\left(a_{i}, r_{i}\right)} u_{\varepsilon}^{2}\right)(|\ln \varepsilon|-C \ln |\ln \varepsilon|)\right]\left|d_{i}\right| \\
& +4 \pi H \sum_{i \in I} d_{i}\left(h_{\varepsilon}-1\right)\left(a_{i}\right)-C H|\ln \varepsilon|^{-4},
\end{aligned}
$$

where $h=\operatorname{curl} A$ and $h_{\varepsilon}$ is introduced in (3.4).

The proof is essentially that of [22], Theorem 5.3.

Let us recall the definition of $\lambda_{\varepsilon}$ in (3.17). We put

$$
k_{\varepsilon}=\frac{1}{2 \lambda_{\varepsilon}}=\frac{1}{2}\left(\max _{x \in \bar{\Omega}}\left|\xi_{\varepsilon}(x)\right|\right)^{-1} .
$$


Corollary 4.3. With the notations of Theorem 4.2, the following lower bound holds:

$$
\begin{aligned}
\mathcal{F}_{\varepsilon, H}(\varphi, A) \geq & H^{2} J_{0}(\varepsilon)+\int_{\Omega \backslash \cup_{i} B\left(a_{i}, r_{i}\right)} \frac{1}{u_{\varepsilon}^{2}}\left|\nabla\left(h-H h_{\varepsilon}\right)\right|^{2} \mathrm{~d} x+\int_{\Omega}\left|h-H h_{\varepsilon}\right|^{2} \mathrm{~d} x \\
& +2 \pi \chi_{\varepsilon}(a) \sum_{d_{i}>0}\left(|\ln \varepsilon|-2 k_{\varepsilon}^{-1} H-C \ln |\ln \varepsilon|\right) d_{i} \\
& +\min (1, a) \sum_{d_{i} \leq 0}(|\ln \varepsilon|-C \ln |\ln \varepsilon|)\left|d_{i}\right|-C H|\ln \varepsilon|^{-4},
\end{aligned}
$$

where $\chi_{\varepsilon}(a)=\min (1, a)$ if $|\ln \varepsilon|-2 k_{\varepsilon}^{-1} H \geq 0$, and $\chi_{\varepsilon}(a)=\max (1, a)$ otherwise.

Proof. Let us assign to each ball $B\left(a_{i}, r_{i}\right)$ a point $a_{i}^{\prime} \in \overline{B\left(a_{i}, r_{i}\right) \cap \Omega}$ such that

$$
u_{\varepsilon}\left(a_{i}^{\prime}\right)=\frac{\min }{B\left(a_{i}, r_{i}\right)} u_{\varepsilon}
$$

Then, thanks to Lemma 3.3 and to the first point of Theorem 4.2, there exists a constant $c>0$ such that

$$
\forall i, \quad\left|h_{\varepsilon}\left(a_{i}\right)-h_{\varepsilon}\left(a_{i}^{\prime}\right)\right| \leq c\left|a_{i}-a_{i}^{\prime}\right| \leq c|\ln \varepsilon|^{-10} .
$$

This permits us to write

$$
\sum_{d_{i}>0}\left[\left(\min _{B\left(a_{i}, r_{i}\right)} u_{\varepsilon}^{2}\right)|\ln \varepsilon|+2 H\left(h_{\varepsilon}\left(a_{i}\right)-1\right)\right] d_{i} \geq \sum_{d_{i}>0} u_{\varepsilon}^{2}\left(a_{i}^{\prime}\right)\left[|\ln \varepsilon|-\left(\frac{1-h_{\varepsilon}\left(a_{i}^{\prime}\right)}{2 u_{\varepsilon}^{2}\left(a_{i}^{\prime}\right)}\right) H-2 c|\ln \varepsilon|^{-4} H\right] d_{i} .
$$

By definition of $k_{\varepsilon}$, we have $\frac{1-h_{\varepsilon}\left(a_{i}^{\prime}\right)}{2 u_{\varepsilon}^{2}\left(a_{i}^{\prime}\right)} \leq k_{\varepsilon}^{-1}$. By Theorem 2.1, $\min (1, a) \leq u_{\varepsilon}^{2}\left(a_{i}^{\prime}\right) \leq \max (1, a)$. Therefore, we get

$$
\sum_{d_{i}>0}\left[\left(\min _{B\left(a_{i}, r_{i}\right)} u_{\varepsilon}^{2}\right)|\ln \varepsilon|+2 H\left(h_{\varepsilon}\left(a_{i}\right)-1\right)\right] d_{i} \geq \chi_{\varepsilon}(a)\left(|\ln \varepsilon|-2 k_{\varepsilon}^{-1} H\right) d_{i} .
$$

For the terms with negative degrees, we write

$$
\sum_{d_{i} \leq 0}\left[\left(\min _{B\left(a_{i}, r_{i}\right)} u_{\varepsilon}^{2}\right)|\ln \varepsilon|-2 H\left(h_{\varepsilon}\left(a_{i}\right)-1\right)\right]\left|d_{i}\right| \geq \min (1, a) \sum_{d_{i} \leq 0}|\ln \varepsilon|\left|d_{i}\right| .
$$

Substituting (4.8)-(4.9) in (4.5), we get the desired lower bound of the corollary.

\subsection{Upper bound on the total degree}

Let us assume from now on that $(\psi, A)$ is a minimizer of $(1.1)$ and that $\left(B\left(a_{i}, r_{i}\right)\right)_{i}$ is its associated family of vortex-balls constructed in Theorem 4.2. Our aim is to give an upper bound on the total degree $\sum_{i}\left|d_{i}\right|$. The answer will be strongly dependent on the parameter $a$.

Lemma 4.4. Assume that for a given constant $K>0$, the magnetic field satisfies $H \leq k_{\varepsilon}|\ln \varepsilon|+K \ln |\ln \varepsilon|$. With the notation of Theorem 4.2, the following two assertions hold.

(1) If $a>1$, then there exists constants $C>0$ and $\left.\left.\varepsilon_{0} \in\right] 0,1\right]$ such that,

$$
\left.\left.\max _{\left|d_{i}\right|>0}\left|a_{i}\right| \leq C|\ln \varepsilon|^{-1 / 4}, \quad \sum_{i}\left|d_{i}\right| \leq C, \quad \forall \varepsilon \in\right] 0, \varepsilon_{0}\right] .
$$


(2) There exists $\left.a_{0} \in\right] 0,1[$ such that, if $\left.a \in] 0, a_{0}\right]$, there exists positive constants $\varepsilon_{0}$ and $C$ such that,

$$
\left.\left.\max _{\left|d_{i}\right|>0}|R-| a_{i}|| \leq C|\ln \varepsilon|^{-1 / 4}, \quad \sum_{i}\left|d_{i}\right| \leq C \ln |\ln \varepsilon|, \quad \forall \varepsilon \in\right] 0, \varepsilon_{0}\right] .
$$

Since the proof of assertion (1) is very close to that of [28], Theorem 2 (with only very few technical modifications), we omit it. We give rather the proof of the second assertion of the lemma (which is also close to that of [28], Thm. 2).

Let us introduce:

$$
D_{+}=\sum_{i, d_{i}>0}\left|d_{i}\right|, \quad D_{-}=\sum_{i, d_{i} \leq 0}\left|d_{i}\right|, \quad D=D_{+}+D_{-}=\sum_{i}\left|d_{i}\right|
$$

and

$$
D_{0}=\sum_{|R-| a_{i}|| \leq|\ln \varepsilon|^{-1 / 4}}\left|d_{i}\right|
$$

We make the following two claims:

$$
\exists C>0, \quad D_{-} \leq C D_{+} \frac{\ln |\ln \varepsilon|}{|\ln \varepsilon|},
$$

and

$$
\exists C>0, \quad D-D_{0} \leq C D \frac{\ln |\ln \varepsilon|}{\sqrt{|\ln \varepsilon|}} .
$$

Now we show that when the claims (4.14) and (4.15) hold, then we can prove assertion (2) of Lemma 4.4.

We put $\widetilde{\Omega}=\Omega \backslash \cup_{i} B\left(a_{i}, r_{i}\right)$, where $B\left(a_{i}, r_{i}\right)$ are the vortex-balls constructed in Theorem 4.2. For a given $t>0$, we denote by $C_{t}$ the circle of center 0 and radius $t$, and by $B_{t}$ the open ball of center 0 and radius $t$. Let us introduce the set of positive real numbers:

$$
E=\{t \in] R+|\ln \varepsilon|^{-1 / 4}, 1\left[: C_{t} \subset \widetilde{\Omega}\right\} .
$$

Thanks to Theorem 4.2, the set $E$ is non empty and has a positive measure

$$
\liminf _{\varepsilon \rightarrow 0}|E|>0 .
$$

Theorem 4.2 gives $|\varphi| \geq 1-|\ln \varepsilon|^{-4}$ on $C_{t}$ whenever $t \in E$. Therefore, the degree

$$
d_{t}=\operatorname{deg}\left(\frac{\varphi}{|\varphi|}, C_{t}\right)
$$

is well defined whenever $t \in E$.

Writing $h=\operatorname{curl} A$ and $\varphi=|\varphi| \mathrm{e}^{\mathrm{i} \phi}$ for an $H^{2}$-function $\phi$, the following equation holds

$$
-\frac{1}{u_{\varepsilon}^{2}} \nabla^{\perp} h=|\varphi|^{2}(\nabla \phi-A) \quad \text { in } \widetilde{\Omega} .
$$

Let us recall also the equation for $h_{\varepsilon}$,

$$
-\operatorname{div}\left(\frac{1}{u_{\varepsilon}^{2}} \nabla h_{\varepsilon}\right)+h_{\varepsilon}=0 \quad \text { in } \Omega
$$


from which it follows, by Stoke's formula:

$$
\int_{C_{t}} \frac{1}{u_{\varepsilon}^{2}} n \cdot \nabla h_{\varepsilon} \mathrm{d} \theta=\int_{B_{t}} h_{\varepsilon} \mathrm{d} x
$$

where $n$ is the unit outward normal vector of $B_{t}, n(x)=x /|x|$ for all $x \in \mathbb{R}^{2} \backslash\{0\}$.

On the other hand, it results from (4.17) and Stoke's formula,

$$
\begin{aligned}
\int_{C_{t}} \frac{1}{u_{\varepsilon}^{2}} n \cdot \nabla h \mathrm{~d} \theta & =\int_{B_{t}}|\varphi|^{2} \tau \cdot(\nabla \phi-A) \mathrm{d} x \\
& =\int_{B_{t}} \tau \cdot \nabla \phi \mathrm{d} x-\int_{C_{t}} h \mathrm{~d} x+T(t),
\end{aligned}
$$

where $(n, \tau)$ is a direct frame, and

$$
T(t)=\int_{B_{t}}\left(|\varphi|^{2}-1\right) \tau \cdot(\nabla \phi-A) \mathrm{d} x .
$$

Coming back to the definition of the degree, we deduce that

$$
\int_{C_{t}} \frac{1}{u_{\varepsilon}^{2}} n \cdot \nabla h \mathrm{~d} \theta=2 \pi d_{t}-\int_{B_{t}} h \mathrm{~d} x+T(t) .
$$

Combining (4.18) and (4.20), we get

$$
\int_{C_{t}} \frac{1}{u_{\varepsilon}^{2}}\left(\nabla h-H \nabla h_{\varepsilon}\right) \cdot n \mathrm{~d} \theta+\int_{B_{t}}\left(h-H h_{\varepsilon}\right) \mathrm{d} x=2 \pi d_{t}+T(t) .
$$

Applying Cauchy-Schwarz inequality on each integral and squaring, we obtain (recall that the function $u_{\varepsilon}$ is radial)

$$
\int_{C_{t}} \frac{1}{u_{\varepsilon}^{2}}\left|\nabla\left(h-H h_{\varepsilon}\right)\right|^{2} \mathrm{~d} \theta+\frac{t}{2} \int_{B_{t}}\left|h-H h_{\varepsilon}\right|^{2} \mathrm{~d} x \geq \frac{\pi}{3 t} d_{t}^{2}-C\left[T^{2}(t)+u_{\varepsilon}^{-2}(t)\right] .
$$

Thanks to (4.14) and (4.15), we infer from the above lower bound

$$
\int_{C_{t}} \frac{1}{u_{\varepsilon}^{2}}\left|\nabla\left(h-H h_{\varepsilon}\right)\right|^{2} \mathrm{~d} \theta+\frac{t}{2} \int_{B_{t}}\left|h-H h_{\varepsilon}\right|^{2} \mathrm{~d} x \geq \frac{\pi}{4 t} D^{2}-C\left[T^{2}(t)+u_{\varepsilon}^{-2}(t)\right],
$$

where $D$ is the total degree introduced in (4.12).

Now, we integrate both sides of (4.21) with respect to $t$ and we recall that inf $E>R$. This yields

$$
\begin{aligned}
\int_{\widetilde{\Omega}} \frac{1}{u_{\varepsilon}^{2}}\left|\nabla\left(h-H h_{\varepsilon}\right)\right|^{2} \mathrm{~d} x+\int_{\Omega}\left|h-H h_{\varepsilon}\right|^{2} \mathrm{~d} x & \geq \int_{E}\left(\int_{C_{t}} \frac{1}{u_{\varepsilon}^{2}}\left|\nabla\left(h-H h_{\varepsilon}\right)\right|^{2} \mathrm{~d} \theta+\frac{t}{2} \int_{B_{t}}\left|h-H h_{\varepsilon}\right|^{2} \mathrm{~d} x\right) \mathrm{d} t \\
& \geq \int_{E}\left(\frac{\pi}{4 t} D^{2}-C\left[T^{2}(t)+u_{\varepsilon}^{-2}(t)\right]\right) \mathrm{d} t \\
& \geq \frac{|E|}{4} D^{2}-C \int_{E}\left(T^{2}(t)+u_{\varepsilon}^{-2}(t)\right) \mathrm{d} t \\
& \geq \widetilde{C}\left[D^{2}-\int_{E}\left(T^{2}(t)+u_{\varepsilon}^{-2}(t)\right) \mathrm{d} t\right],
\end{aligned}
$$

where $\widetilde{C}>0$ is an explicit constant. 
Since $u_{\varepsilon}^{2}>a$ when $a<1$ (see Thm. 2.1), it is clear that $\int_{E} u_{\varepsilon}^{-2} \mathrm{~d} t \leq a^{-1}|E| \leq C$. Let us estimate the integral of $T^{2}(t)$. Notice that

$$
\int_{E} T^{2}(t) \mathrm{d} t \leq \int_{\widetilde{\Omega}}\left(1-|\varphi|^{2}\right)|(\nabla-i A) \varphi|^{2} \mathrm{~d} x \leq|\ln \varepsilon|^{-4} \int_{\Omega}|(\nabla-i A) \varphi|^{2} \mathrm{~d} x \ll 1
$$

where we have used Theorem 4.2 and the constraint on the applied magnetic field $H=\mathcal{O}(|\ln \varepsilon|)$.

Therefore, we conclude finally that, for a possibly larger explicit constant $\widetilde{C}>0$,

$$
\int_{\widetilde{\Omega}} \frac{1}{u_{\varepsilon}^{2}}\left|\nabla\left(h-H h_{\varepsilon}\right)\right|^{2} \mathrm{~d} x+\int_{\Omega}\left|h-H h_{\varepsilon}\right|^{2} \mathrm{~d} x \geq \widetilde{C}\left(D^{2}-1\right) .
$$

We substitute (4.14), (4.15) and (4.22) in (4.5) to obtain:

$$
\mathcal{F}_{\varepsilon, H}(\varphi, A) \geq H^{2} J_{0}(\varepsilon)+\widetilde{C}\left(D^{2}-1\right)-C \ln |\ln \varepsilon| D
$$

Matching this lower bound with the upper bound (4.1), we deduce that

$$
D^{2} \leq C^{\prime} \ln |\ln \varepsilon| D
$$

which permits us to deduce the statement concerning the total degree in the second assertion of Lemma 4.4 . Substituting the bound of $D$ in (4.14) and (4.15), we deduce that

$$
D_{-}=0, \quad D_{0}=D
$$

thus proving that all the vortices have positive degrees together with the first statement in the assertion (2) of Lemma 4.4.

We have only to prove claims (4.14) and (4.15). Claim (4.14) is a direct consequence of Theorem 4.2. Claim (4.15) is a consequence of Lemma 3.5.

\section{UPPER BOUND OF THE ENERGY}

\subsection{Main result}

In this section, we assume that the magnetic field satisfies

$$
H=k_{\varepsilon}|\ln \varepsilon|+\lambda \ln |\ln \varepsilon|, \quad(\lambda \in \mathbb{R}),
$$

where $k_{\varepsilon}$ is introduced in (4.6).

The aim of this section is to establish the following upper bound for the energy $\mathcal{F}_{\varepsilon, H}(\varphi, A)$, where the functional $\mathcal{F}_{\varepsilon, H}$ is introduced in (2.7). Let us recall the constant $\left.a_{0} \in\right] 0,1[$ introduced in Theorem 3.4.

Proposition 5.1. Let $(\psi, A)$ be a minimizer of $(1.1)$ and $\varphi=\frac{\psi}{u_{\varepsilon}}$. Assume that a $\left.\in\right] 0, a_{0}[$. There exist constants $C_{*}>0, \varepsilon_{0}>0$ such that, when the applied magnetic field $\stackrel{H}{H}$ satisfies (5.1), the following upper bound of the energy holds,

$$
\left.\left.\mathcal{F}_{\varepsilon}(\varphi, A) \leq H^{2} J_{0}(\varepsilon)+\left(C_{*}-\lambda\right)(\ln |\ln \varepsilon|)^{2}, \quad \forall \varepsilon \in\right] 0, \varepsilon_{0}\right]
$$

The proof of Proposition 5.1 is by constructing a suitable test configuration having vortices and by computing its energy. The estimate of the energy of the test configuration relies on a careful analysis of a Green's potential. 


\subsection{Analysis of a Green's potential}

This section is devoted to an analysis of a Green's kernel, i.e. a fundamental solution of the differential operator $-\operatorname{div}\left(\frac{1}{u_{\varepsilon}^{2}(x)} \nabla\right)+1$. The existence and the properties of this function, taken from $[1,33]$, are given in the next lemma.

Lemma 5.2. For every $y \in \Omega$ and $\varepsilon \in] 0,1]$, there exists a symmetric function $\bar{\Omega} \times \bar{\Omega} \ni(x, y) \mapsto G_{\varepsilon}(x, y) \in \mathbb{R}_{+}$ such that:

$$
\left\{\begin{aligned}
-\operatorname{div}\left(\frac{1}{u_{\varepsilon}^{2}(x)} \nabla_{x} G_{\varepsilon}(x, y)\right)+G_{\varepsilon}(x, y) & =\delta_{y}(x) \text { in } \mathcal{D}^{\prime}(\Omega), \\
\left.G_{\varepsilon}(x, y)\right|_{x \in \partial \Omega} & =0 .
\end{aligned}\right.
$$

Moreover, $G_{\varepsilon}$ satisfies the following properties:

(1) There exists a constant $C>0$ such that

$$
\left.\left.G_{\varepsilon}(x, y) \leq C(|\ln | x-y||+1), \quad \forall(x, y) \in \bar{\Omega} \times \bar{\Omega} \backslash \Delta, \forall \varepsilon \in\right] 0,1\right],
$$

where $\Delta$ denotes the diagonal in $\mathbb{R}^{2}$.

(2) The function $v_{\varepsilon}: \bar{\Omega} \times \bar{\Omega} \ni(x, y) \mapsto G_{\varepsilon}(x, y)+\frac{u_{\varepsilon}^{2}(x)}{2 \pi} \ln |x-y|$ is in the class $C^{1}(\bar{\Omega} \times \bar{\Omega} ; \mathbb{R})$.

(3) Given $q \in[1,2[$, there exists a constant $C>0$ such that

$$
\left.\left.\left\|v_{\varepsilon}(\cdot, y)\right\|_{W^{1, q}(\Omega)} \leq C, \quad \forall y \in \bar{\Omega}, \quad \forall \varepsilon \in\right] 0,1\right] .
$$

(4) For any compact set $K \subset \Omega$, there exist constants $C>0$ and $\varepsilon_{0}>0$ such that, $\left.\left.\forall \varepsilon \in\right] 0, \varepsilon_{0}\right]$,

$$
\left|G_{\varepsilon}(x, y)+\frac{u_{\varepsilon}^{2}(x)}{2 \pi} \ln \right| x-y|| \leq C\left\|\nabla u_{\varepsilon}(x)\right\|_{L^{\infty}(K)}, \quad \forall x \in K, \forall y \in \bar{\Omega} .
$$

Corollary 5.3. Assume that $a \in] 0, a_{0}\left[\right.$ and $\left.R^{\prime} \in\right] R, 1[$. There exist constants $C>0, \alpha \in] 0,1\left[\right.$ and $\varepsilon_{0}>0$ such that, for all $\varepsilon \in] 0, \varepsilon_{0}\left[\right.$ and $2\left(R_{\varepsilon}-R\right)<\eta(\varepsilon)<1$, we have

$$
\left\|v_{\varepsilon}(\cdot, y)\right\|_{C^{0, \alpha}\left(D\left(0, R^{\prime}\right) \backslash D(0, R+\eta(\varepsilon))\right)} \leq \frac{C}{\eta(\varepsilon)^{2}}, \quad \forall y \in \bar{\Omega} .
$$

Here

$$
v_{\varepsilon}(x, y)=G_{\varepsilon}(x, y)+\frac{u_{\varepsilon}^{2}(x)}{2 \pi} \ln |x-y| .
$$

Proof. Let $\chi \in C_{0}^{\infty}(\mathbb{R} ; \mathbb{R})$ be a cut-off function such that

$$
0 \leq \chi \leq 1 \quad \text { in } \mathbb{R}, \quad \chi \equiv 1 \quad \text { in }\left[1, \infty[, \quad \chi \equiv 0 \quad \text { in }]-\infty, \frac{1}{2}[.\right.
$$

Set

$$
\chi_{\eta}(x)=\chi\left(\frac{|x|}{\eta}\right), \quad \widetilde{v}_{\varepsilon}(x)=\chi_{\eta}(x) v_{\varepsilon}(x), \quad \forall x \in D(0,1) .
$$

The function $\widetilde{v}_{\varepsilon}$ satisfies the equation

$$
-\operatorname{div}\left(\frac{1}{u_{\varepsilon}^{2}} \nabla_{x} \widetilde{v}_{\varepsilon}\right)+\widetilde{v}_{\varepsilon}(x, y)=\chi_{\eta} f_{\varepsilon}(x, y)+w_{\varepsilon}(x, y),
$$


where

and

$$
f_{\varepsilon}(x, y)=\frac{u_{\varepsilon}^{2}(y)}{\pi u_{\varepsilon}^{3}(x)} \nabla u_{\varepsilon}(x) \cdot \nabla_{x} \ln |x-y|-\frac{u_{\varepsilon}^{2}(y)}{2 \pi} \ln |x-y|,
$$

$$
w_{\varepsilon}(x, y)=\frac{\nabla_{x} v_{\varepsilon}(x, y) \cdot \nabla \chi_{\eta}(x)}{u_{\varepsilon}^{2}(x)}-2 \frac{v_{\varepsilon}(x, y)}{u_{\varepsilon}^{3}(x)} \nabla u_{\varepsilon}(x) \cdot \nabla \chi_{\eta}(x)+\frac{v_{\varepsilon}(x, y)}{u_{\varepsilon}^{2}(x)} \Delta \chi_{\eta}(x) .
$$

Let us notice also that it results from Theorems 2.1 and 3.4

$$
\left\|\nabla u_{\varepsilon}\right\|_{L^{\infty}(D(0,1) \backslash D(0, R+\eta))} \leq C, \quad u_{\varepsilon} \geq \sqrt{a} \text { in } D(0,1) .
$$

Thanks to the above properties of the function $u_{\varepsilon}$, we deduce that for a given $q \in[1,2[$, there exists a constant $C>0$ such that

$$
\left.\left.\left\|\chi_{\eta} f_{\varepsilon}(\cdot, y)\right\|_{L^{q}(D(0,1))} \leq C, \quad \forall y \in \bar{\Omega}, \forall \varepsilon \in\right] 0,1\right] .
$$

On the other hand, for a given $q \in\left[1,2\left[\right.\right.$, it is known that the function $v_{\varepsilon}(\cdot, y)$ is bounded in $W^{1, q}(\Omega)$ (see Lem. 5.2). Thus, we get the following estimate for the functions $\widetilde{v}_{\varepsilon}$ and $w_{\varepsilon}$ :

$$
\left.\left.\left\|\widetilde{v}_{\varepsilon}(\cdot, y)\right\|_{W^{1, q}(D(0,1))} \leq C, \quad\left\|w_{\varepsilon}(\cdot, y)\right\|_{L^{q}(D(0,1))} \leq \frac{C}{\eta^{2}}, \forall y \in \overline{D(0,1)}, \forall \varepsilon \in\right] 0,1\right] .
$$

Let $\left.R^{\prime} \in\right] R, 1\left[\right.$. Thanks to the equation of $\widetilde{v}_{\varepsilon}$, Theorem 2 of [25] implies that there exist $p>2$ and $p^{\prime}<2$ such that

$$
\left\|\nabla \widetilde{v}_{\varepsilon}(\cdot, y)\right\|_{L^{p}\left(D\left(0, R^{\prime}\right)\right)} \leq C\left(\left\|\nabla \widetilde{v}_{\varepsilon}(\cdot, y)\right\|_{L^{p^{\prime}}(D(0,1))}+\left\|w_{\varepsilon}(\cdot, y)+\chi_{\eta} f_{\varepsilon}(\cdot, y)\right\|_{W^{-1, p}(D(0,1))}\right) .
$$

We may choose $q \in] 1,2\left[\right.$ such that $L^{q}$ is embedded in $W^{-1, p}$ and $p^{\prime}<q$. Thus, the above estimate reads as:

$$
\left\|\nabla \widetilde{v}_{\varepsilon}(\cdot, y)\right\|_{L^{p}\left(D\left(0, R^{\prime}\right)\right)} \leq \frac{C}{\eta^{2}}, \quad \forall y \in \overline{D(0,1)} .
$$

Since $\widetilde{v}_{\varepsilon}$ is bounded uniformly in $W^{1, q}(\Omega)$ (see Lem. 5.2), we get by Poincaré's inequality:

$$
\left\|\widetilde{v}_{\varepsilon}(\cdot, y)\right\|_{W^{1, p}\left(D\left(0, R^{\prime}\right)\right)} \leq \frac{C}{\eta^{2}}, \quad \forall y \in \overline{D(0,1)}
$$

Since $p>2$, the Sobolev embedding theorem yields the bound

$$
\left\|\widetilde{v}_{\mathcal{\varepsilon}}(\cdot, y)\right\|_{C^{0, \alpha}\left(D\left(0, R^{\prime}\right)\right)} \leq \frac{C}{\eta^{2}}, \quad \forall y \in \overline{D(0,1)},
$$

for some $\alpha \in] 0,1[$. This estimate is nothing but the result of the corollary once we remember the definition of the function $\widetilde{v}_{\varepsilon}$.

The next lemma provides us with points enjoying useful properties. These points will serve to be the centers of the vortices of the test configuration that we shall construct in the next section.

Lemma 5.4. There exist constants $K>0, c \in] 0,1[$, and for each $\varepsilon \in] 0,1\left[\right.$ and $n(\varepsilon) \in \mathbb{N} \cap\left[1, \frac{c}{2} \varepsilon^{-1}[\right.$, there exist points $\left(a_{i}\right)_{i=1}^{n(\varepsilon)} \subset \partial D\left(0, r_{\varepsilon}\right)$ and $\left.\delta(\varepsilon) \in\right] 0,1[$ such that

$$
\begin{aligned}
& \delta(\varepsilon) \ll 1 \quad \text { as } \varepsilon \rightarrow 0 \\
& \frac{c}{n(\varepsilon)} \leq\left|a_{i+1}-a_{i}\right| \leq \delta(\varepsilon)+\frac{c}{n(\varepsilon)}, \quad\left|v_{\varepsilon}\left(a_{i}, a_{i}\right)\right| \leq K \ln |\ln \varepsilon|, \\
& \forall i \in\{1,2, \ldots, n(\varepsilon)\}, \quad \forall \varepsilon \in] 0,1] .
\end{aligned}
$$

Here the function $v_{\varepsilon}$ has been introduced in Lemma 5.2 , and $r_{\varepsilon}=R+\frac{\ln |\ln \varepsilon|}{|\ln \varepsilon|}$. 
Proof. The proof is actually due to the following bound

$$
\int_{\partial D\left(0, r_{\varepsilon}\right)}\left|v_{\varepsilon}(x, x)\right| \mathrm{d} x \leq C \ln |\ln \varepsilon|,
$$

that holds uniformly in $\varepsilon \in] 0,1]$. Let us show why this bound holds. We cover $\partial D\left(0, r_{\varepsilon}\right)$ by $\mathcal{N}$ balls $\left(B\left(y_{i}, \zeta\right)\right)_{i}$, with $\left(y_{i}\right)_{i} \subset \partial D\left(0, r_{\varepsilon}\right)$ and $\left.\zeta \in\right] 0,1\left[\right.$ is to be chosen appropriately. We introduce a scaled partition of unity $\chi_{i}^{\zeta}$ such that

$$
\sum_{i=1}^{\mathcal{N}}\left|\chi_{i}^{\zeta}\right|=1 \text { in } \partial D\left(0, r_{\varepsilon}\right), \quad \operatorname{supp} \chi_{i}^{\zeta} \subset B\left(y_{i}, \zeta\right), \quad \forall i \in\{1, \ldots, \mathcal{N}\} .
$$

Then

$$
\int_{\partial D\left(0, r_{\varepsilon}\right)}\left|v_{\varepsilon}(x, x)\right| \mathrm{d} x=\sum_{i=1}^{\mathcal{N}} \int_{\partial D\left(0, r_{\varepsilon}\right)}\left|\chi_{i}^{\zeta}(x) v_{\varepsilon}(x, x)\right| \mathrm{d} x .
$$

By Corollary 5.3, we write for all $i \in\{1, \ldots, \mathcal{N}\}$

$$
\int_{\partial D\left(0, r_{\varepsilon}\right)}\left|\chi_{i}^{\zeta}(x) v_{\varepsilon}(x, x)\right| \mathrm{d} x \leq \int_{\partial D\left(0, r_{\varepsilon}\right)}\left|\chi_{i}^{\zeta}(x) v_{\varepsilon}\left(x, y_{i}\right)\right| \mathrm{d} \mu_{*}(x)+\frac{C}{\eta^{2}} \int_{\partial D\left(0, r_{\varepsilon}\right)}\left|\chi_{i}^{\zeta}(x)\right|\left|x-y_{i}\right|^{\alpha} \mathrm{d} \mu_{*}(x),
$$

where $\alpha \in] 0,1\left[, \eta=R-r_{\varepsilon}\right.$, and $\mu_{*}$ is the Lebesgue measure in $\partial D\left(0, r_{\varepsilon}\right)$.

Recalling that supp $\chi_{i}^{\zeta} \subset B\left(y_{i}, \zeta\right)$, we get upon choosing $\zeta=\eta^{2 / \alpha}$ and summing up over $i$,

$$
\sum_{i=1}^{\mathcal{N}} \frac{1}{\eta^{2}} \int_{\partial D\left(0, r_{\varepsilon}\right)}\left|\chi_{i}^{\zeta}(x)\right|\left|x-y_{i}\right|^{\alpha} \mathrm{d} \mu_{*}(x) \leq C .
$$

On the other hand, by Lemma 5.2, there exists a constant $C>0$ such that

$$
\begin{aligned}
\int_{\partial D\left(0, r_{\varepsilon}\right)}\left|\chi_{i}^{\zeta}(x) v_{\varepsilon}\left(x, y_{i}\right)\right| \mathrm{d} \mu_{*}(x) & \leq C \int_{B\left(y_{i}, \zeta\right) \cap \partial D\left(0, r_{\varepsilon}\right)}|\ln | x-y_{i}|| \mathrm{d} \mu_{*}(x) \\
& \leq C \zeta|\ln \zeta| .
\end{aligned}
$$

Recalling our choice of $\zeta=\eta^{2 / \alpha}$ and $\eta=\mathcal{O}\left(|\ln \varepsilon|^{-1 / 2}\right)$, and summing up over $i$, we get for a new constant $C>0$

$$
\sum_{i=1}^{\mathcal{N}} \int_{\partial D\left(0, r_{\varepsilon}\right)}\left|\chi_{i}^{\zeta}(x) v_{\varepsilon}(x, x)\right| \mathrm{d} x \leq C \mathcal{N} \times \zeta \ln |\ln \varepsilon| \leq C \ln |\ln \varepsilon|,
$$

where we have used that $\mathcal{N} \times 2 \pi \zeta \approx 2 \pi r_{\varepsilon} \rightarrow 2 \pi R$. Substituting in (5.5), we obtain the desired bound (5.4).

Now, defining the function

$$
f_{\varepsilon}(t):\left[0,1\left[\ni t \mapsto\left|v_{\varepsilon}\left(r_{\varepsilon} \mathrm{e}^{2 \pi \mathrm{i} t}, r_{\varepsilon} \mathrm{e}^{2 \pi \mathrm{i} t}\right)\right|,\right.\right.
$$

and applying Lemma 5.5 below, we get the desired sequence of points.

Lemma 5.5. Let $\left(f_{\varepsilon}\right)_{\varepsilon \in] 0,1]} \subset C\left([0,1], \mathbb{R}_{+}\right)$be a family of continuous functions. Assume that there exists a constant $C>0$ such that

$$
\left.\left.\left\|f_{\varepsilon}\right\|_{L^{1}([0,1])} \leq C \ln |\ln \varepsilon|, \quad \forall \varepsilon \in\right] 0,1\right] .
$$

There exist constants $K>0$ and $\left.c_{0} \in\right] 0,1[$ such that, given a family $(N(\varepsilon)) \subset \mathbb{N}$ satisfying $N(\varepsilon) \gg 1$, there exists a family $(\delta(\varepsilon)) \subset] 0,1\left[\right.$ and a sequence $\left.\left(t_{m}^{\varepsilon}\right)_{m \in \mathbb{N}} \subset\right] 0,1[$ and

$$
\begin{aligned}
& \left|f_{\varepsilon}\left(t_{i}^{\varepsilon}\right)\right| \leq K \ln |\ln \varepsilon|, \quad \frac{c_{0}}{N(\varepsilon)} \leq\left|t_{i+1}^{\varepsilon}-t_{i}^{\varepsilon}\right| \leq \delta(\varepsilon)+\frac{c_{0}}{N(\varepsilon)}, \\
& \forall i \in\{1,2, \ldots, N(\varepsilon)\}, \quad \forall \varepsilon \in] 0,1] .
\end{aligned}
$$


Proof. Let us introduce, for a given $K>0$, the set

$$
E_{K}^{\varepsilon}=\left\{t \in[0,1]:\left|f_{\varepsilon}(t)\right|<K \ln |\ln \varepsilon|\right\} .
$$

Using the uniform bound on $\left\|f_{\varepsilon}\right\|_{L^{1}([0,1])}$, we can choose $K$ sufficiently large such that

$$
\left.\left.\left|E_{K}^{\varepsilon}\right| \geq \frac{1}{2} \quad \forall \varepsilon \in\right] 0,1\right]
$$

where $|\cdot|$ denotes the Lebesgue measure.

Let $\varepsilon \in] 0,1]$. Since the function $f_{\varepsilon}$ is continuous, the set $E_{K}^{\varepsilon}$ is open. Thus, we essentially meet two cases: Either there exists an interval

$$
] x_{\varepsilon}-\delta_{\varepsilon}, x_{\varepsilon}+\delta_{\varepsilon}\left[\subset E_{K}^{\varepsilon}\right.
$$

with $\liminf _{\varepsilon \rightarrow 0} \delta_{\varepsilon}>0$ (in which case the statement of the theorem becomes evidently true), or there exists a constant $\left.c_{0} \in\right] 0, \frac{1}{2}[$ and possibly infinitely many disjoint intervals

$$
\bigcup_{i \in I_{\varepsilon}} x_{i}^{\varepsilon}-\delta_{i}^{\varepsilon}, x_{i}^{\varepsilon}+\delta_{i}^{\varepsilon}\left[\subset E_{K}^{\varepsilon}\right.
$$

such that $x_{1}^{\varepsilon}<x_{2}^{\varepsilon}<\cdots$ and

$$
\limsup _{\varepsilon \rightarrow 0}\left(\sum_{i \in I_{\varepsilon}} \Delta_{i}\right)=0, \quad \liminf _{\varepsilon \rightarrow 0}\left(\sum_{i \in I_{\varepsilon}} \delta_{i}^{\varepsilon}\right) \geq \frac{c_{0}}{2},
$$

where $\Delta_{i}=\left|x_{i+1}^{\varepsilon}-\delta_{i+1}^{\varepsilon}-x_{i}^{\varepsilon}-\delta_{i}^{\varepsilon}\right|$.

Consequently, setting $t_{1}^{\varepsilon}=x_{1}^{\varepsilon}$ and $\left.\mathcal{E}_{K}^{\varepsilon}=\bigcup_{i \in I_{\varepsilon}}\right] x_{i}^{\varepsilon}-\delta_{i}^{\varepsilon}, x_{i}^{\varepsilon}+\delta_{i}^{\varepsilon}$, we get

$$
\mathcal{E}_{K}^{\varepsilon} \backslash t_{1}^{\varepsilon}, t_{1}^{\varepsilon}+\frac{c_{0}}{8 N(\varepsilon)}[\neq \emptyset
$$

So, setting

$$
t_{2}^{\varepsilon}=\inf \left(\mathcal{E}_{K}^{\varepsilon} \backslash t_{1}^{\varepsilon}, t_{1}^{\varepsilon}+\frac{c_{0}}{8 N(\varepsilon)}[)\right.
$$

we get, thanks in particular to (5.6),

$$
t_{2}^{\varepsilon}-t_{1}^{\varepsilon} \leq 2 \sum_{i} \Delta_{i}+\frac{c_{0}}{8 N(\varepsilon)}
$$

and

Therefore, we set

$$
\mathcal{E}_{K}^{\varepsilon} \backslash t_{1}^{\varepsilon}, t_{2}^{\varepsilon}+\frac{c_{0}}{8 N(\varepsilon)}[\neq \emptyset \text {. }
$$

$$
t_{3}^{\varepsilon}=\inf \left(\mathcal{E}_{K}^{\varepsilon} \backslash\right] t_{1}^{\varepsilon}, t_{2}^{\varepsilon}+\frac{c_{0}}{8 N(\varepsilon)}[) .
$$

By induction, given $n \leq N(\varepsilon)$, we can construct points $t_{2}^{\varepsilon}<t_{3}^{\varepsilon}<\cdots<t_{n}^{\varepsilon}$ such that

$$
\frac{c_{0}}{8 N(\varepsilon)} \leq t_{i+1}^{\varepsilon}-t_{i}^{\varepsilon} \leq \frac{c_{0}}{8 N(\varepsilon)}+\sum_{i} \Delta_{i}, \quad \forall i \in\{1, \ldots, n\}
$$

yielding therefore the desired sequence $\left(t_{m}^{\varepsilon}\right)$ with $\delta(\varepsilon)=\sum_{i \in I_{\varepsilon}} \Delta_{i}$. 


\subsection{The test configuration}

We know from Theorem 3.4 that the function $\xi_{\varepsilon}(x)$ achieves its unique minimum on the circle $\partial D\left(0, R_{\varepsilon}\right)$ with $\varepsilon \ll R_{\varepsilon}-R \ll \varepsilon^{\alpha}$, for $\left.\alpha \in\right] 0,1[$.

Since we expect vortices of a minimizer of (1.1) to be pinned on the circle $\partial D\left(0, R_{\varepsilon}\right)$, and to be uniformly distributed along this circle, we construct a test configuration whose vortices are placed, for technical reasons, on the circle $\partial D\left(0, r_{\varepsilon}\right)$, with $r_{\varepsilon}=R+\frac{\ln |\ln \varepsilon|}{|\ln \varepsilon|}$. We mention that similar constructions have been also introduced in the papers [2-4].

Let $n(\varepsilon) \in \mathbb{N} \cap] 1, \frac{c}{2} \varepsilon^{-1}[$ for an appropriate constant $c \in] 0,1[$. Lemma 5.4 provides us with $n(\varepsilon)$ points $\left(a_{i}\right)_{i=1}^{n(\varepsilon)}$ on the circle $\partial D\left(0, r_{\varepsilon}\right)$, that satisfy in particular

$$
\overline{B\left(a_{i}, \varepsilon\right)} \cap \overline{B\left(a_{j}, \varepsilon\right)}=\emptyset, \quad \forall i \neq j
$$

We define a measure $\mu$ by:

$$
\mu(x)= \begin{cases}0 & \text { if } x \notin \cup_{i} B\left(a_{i}, \varepsilon\right) \\ \frac{2}{\varepsilon^{2}} & \text { if } x \in \overline{\cup_{i} B\left(a_{i}, \varepsilon\right)}\end{cases}
$$

and a function $h^{\prime}$ in $\Omega=D(0,1)$ by

$$
\left\{\begin{aligned}
-\operatorname{div}\left(\frac{1}{u_{\varepsilon}^{2}} \nabla h^{\prime}\right)+h^{\prime} & =\mu \text { in } \Omega \\
h^{\prime} & =0 \text { on } \partial \Omega
\end{aligned}\right.
$$

We notice that

$$
\int_{B\left(a_{i}, \varepsilon\right)} \mu \mathrm{d} x=2 \pi, \quad \forall i=1,2, \ldots, n(\varepsilon), \quad \int_{\mathbb{R}^{2}} \mu \mathrm{d} x=2 \pi n(\varepsilon) .
$$

We define an induced magnetic field $h=h^{\prime}+h_{\varepsilon}$ (here $h_{\varepsilon}$ has been introduced in (3.4)). Then we define an induced magnetic potential $A=A^{\prime}+\frac{H}{u_{\varepsilon}^{2}} \nabla^{\perp} h_{\varepsilon}$ by taking simply

$$
\operatorname{curl} A^{\prime}=h^{\prime}
$$

This choice is always possible as one can take $A^{\prime}=\nabla^{\perp} g$ with $g \in H^{2}(\Omega)$ such that $\Delta g=h^{\prime}$.

We turn now to define an order parameter $\psi$ which we take in the form

$$
\psi=u u_{\varepsilon}=\rho \mathrm{e}^{\mathrm{i} \phi} u_{\varepsilon}
$$

where $\rho$ is defined by:

$$
\rho(x)=\left\{\begin{array}{cc}
0 & \text { if } x \in \cup_{i} B\left(a_{i}, \varepsilon\right), \\
1 & \text { if } x \notin \cup_{i} B\left(a_{i}, 2 \varepsilon\right), \\
\frac{\left|x-a_{i}\right|}{\varepsilon}-1 & \text { if } \exists i \text { s.t. } x \in B\left(a_{i}, 2 \varepsilon\right) \backslash B\left(a_{i}, \varepsilon\right) .
\end{array}\right.
$$

The phase $\phi$ is defined (modulo $2 \pi$ ) by the relation:

$$
\nabla \phi-A^{\prime}=-\frac{1}{u_{\varepsilon}^{2}} \nabla^{\perp} h^{\prime} \quad \text { in } \Omega \backslash \cup_{i} B\left(a_{i}, \varepsilon\right),
$$

and we emphasize here that we do not need to define $\phi$ in regions where $\rho$ vanishes. 
Lemma 5.6. There exist constants $\left.\varepsilon_{0} \in\right] 0,1[$ and $C>0$ such that

$$
\int_{\Omega}\left(\frac{1}{u_{\varepsilon}^{2}(x)}\left|\nabla h^{\prime}\right|^{2}+\left|h^{\prime}\right|^{2}\right) \mathrm{d} x \mathrm{~d} y \leq 2 \pi u_{\varepsilon}^{2}\left(r_{\varepsilon}\right) n(\varepsilon)|\ln \varepsilon|+C n(\varepsilon) \ln |\ln \varepsilon|+C[n(\varepsilon)]^{2}+o\left([n(\varepsilon)]^{2}\right) .
$$

Proof. Notice that the field $h^{\prime}$ can be expressed by means of the function $G_{\varepsilon}$ introduced in Lemma 5.2,

$$
h^{\prime}(x)=\int_{\Omega} G_{\varepsilon}(x, y) \mu(y) \mathrm{d} y, \quad \forall x \in \Omega .
$$

Therefore, we get the identity

$$
\int_{\Omega}\left(\frac{1}{u_{\varepsilon}^{2}(x)}\left|\nabla h^{\prime}\right|^{2}+\left|h^{\prime}\right|^{2}\right) \mathrm{d} x=\int_{\Omega \times \Omega} G_{\varepsilon}(x, y) \mu(x) \mu(y) \mathrm{d} x \mathrm{~d} y
$$

which shows that it is sufficient to estimate $\int_{\Omega \times \Omega} G_{\varepsilon}(x, y) \mu(x) \mu(y) \mathrm{d} x \mathrm{~d} y$. We decompose the integral $\int_{\Omega \times \Omega} G_{\varepsilon}(x, y) \mu(x) \mu(y) \mathrm{d} x \mathrm{~d} y$ into two terms:

$$
\begin{aligned}
\int_{\Omega \times \Omega} G_{\varepsilon}(x, y) \mu(x) \mu(y) \mathrm{d} x \mathrm{~d} y= & \sum_{i \neq j} \int_{B\left(a_{i}, \varepsilon\right) \times B\left(a_{j}, \varepsilon\right)} G_{\varepsilon}(x, y) \mu(x) \mu(y) \mathrm{d} x \mathrm{~d} y \\
& +\sum_{i=1}^{n(\varepsilon)} \int_{B\left(a_{i}, \varepsilon\right) \times B\left(a_{i}, \varepsilon\right)} G_{\varepsilon}(x, y) \mu(x) \mu(y) \mathrm{d} x \mathrm{~d} y .
\end{aligned}
$$

Let us estimate the first term. We write using Lemma 5.2,

$$
\sum_{i \neq j} \int_{B\left(a_{i}, \varepsilon\right) \times B\left(a_{j}, \varepsilon\right)} G_{\varepsilon}(x, y) \mu(x) \mu(y) \mathrm{d} x \mathrm{~d} y \leq C \sum_{i \neq j} \int_{B\left(a_{i}, \varepsilon\right) \times B\left(a_{j}, \varepsilon\right)}(|\ln | x-y||+1) \mu(x) \mu(y) \mathrm{d} x \mathrm{~d} y .
$$

Now, recalling the definition of $\mu$ in (5.7) and the choice of the points $\left(a_{i}\right)$ in Lemma 5.4 , we get

$$
\sum_{i \neq j} \frac{c}{n(\varepsilon)^{2}} \int_{B\left(a_{i}, \varepsilon\right) \times B\left(a_{j}, \varepsilon\right)}|\ln | x-y|| \mu(x) \mu(y) \mathrm{d} x \mathrm{~d} y \leq C,
$$

where $C>0$ is any constant such that

$$
C>\int_{\partial D(0, R) \times \partial D(0, R)}|\ln | x-y|| \mathrm{d} \mu_{*}(x) \mathrm{d} \mu_{*}(y)
$$

and $\mathrm{d} \mu_{*}$ is the arc-length measure on the circle $\partial D(0, R)$.

Therefore, (5.15) becomes for a new constant $C>0$,

$$
\sum_{i \neq j} \int_{B\left(a_{i}, \varepsilon\right) \times B\left(a_{j}, \varepsilon\right)} G_{\varepsilon}(x, y) \mu(x) \mu(y) \mathrm{d} x \mathrm{~d} y \leq C n(\varepsilon)^{2} .
$$

Again, using Corollary 5.3, we estimate

$$
\int_{B\left(a_{i}, \varepsilon\right) \times B\left(a_{i}, \varepsilon\right)} G_{\varepsilon}(x, y) \mu(x) \mu(y) \mathrm{d} x \mathrm{~d} y=\frac{4}{\varepsilon^{4}} \int_{B\left(a_{i}, \varepsilon\right) \times B\left(a_{i}, \varepsilon\right)}\left(\frac{u_{\varepsilon}^{2}(x)}{2 \pi} \ln \frac{1}{|x-y|}+\left|v_{\varepsilon}(x, y)\right|\right) \mathrm{d} x \mathrm{~d} y .
$$


On the one hand, we have

$$
\frac{4}{\varepsilon^{4}} \int_{B\left(a_{i}, \varepsilon\right) \times B\left(a_{i}, \varepsilon\right)} \frac{u_{\varepsilon}^{2}(x)}{2 \pi} \ln \frac{1}{|x-y|} \mathrm{d} x \mathrm{~d} y=4 \int_{B(0,1) \times B(0,1)} \frac{u_{\varepsilon}^{2}\left(a_{i}+\frac{z^{\prime}}{\varepsilon}\right)}{2 \pi} \ln \left[\frac{1}{\varepsilon\left|z^{\prime}-w^{\prime}\right|}\right] \mathrm{d} z^{\prime} \mathrm{d} w^{\prime} .
$$

Recall that the function $u_{\varepsilon}$ is radial and that $\left|a_{i}\right|=r_{\varepsilon}$. By Theorem 3.4, we have

$$
\left|u_{\varepsilon}^{2}\left(a_{i}+\frac{z^{\prime}}{\varepsilon}\right)-u_{\varepsilon}^{2}\left(r_{\varepsilon}\right)\right| \leq \mathcal{O}(\varepsilon), \quad \forall z^{\prime} \in B(0,1) .
$$

Therefore,

$$
\begin{aligned}
\int_{B\left(a_{i}, \varepsilon\right) \times B\left(a_{i}, \varepsilon\right)} \frac{u_{\varepsilon}^{2}(x)}{2 \pi} \ln \frac{1}{|x-y|} \mu(x) \mu(y) \mathrm{d} x \mathrm{~d} y & \leq 4 \int_{B(0,1) \times B(0,1)} \frac{u_{\varepsilon}^{2}\left(r_{\varepsilon}\right)+\mathcal{O}(\varepsilon)}{2 \pi} \ln \left[\frac{1}{\varepsilon\left|z^{\prime}-w^{\prime}\right|}\right] \mathrm{d} z^{\prime} \mathrm{d} w^{\prime} \\
& =2 \pi u_{\varepsilon}^{2}\left(r_{\varepsilon}\right)|\ln \varepsilon|+o(1) .
\end{aligned}
$$

On the other hand, assuming that the following estimate holds

$$
\limsup _{\varepsilon \rightarrow 0} \frac{1}{n(\varepsilon) \ln |\ln \varepsilon|} \sum_{i=1}^{n(\varepsilon)} \frac{4}{\varepsilon^{4}} \int_{B\left(a_{i}, \varepsilon\right) \times B\left(a_{i}, \varepsilon\right)}\left|v_{\varepsilon}(x, y)\right| \mathrm{d} x \mathrm{~d} y \leq C,
$$

then (5.18) becomes

$$
\sum_{i=1}^{n(\varepsilon)} \int_{B\left(a_{i}, \varepsilon\right) \times B\left(a_{i}, \varepsilon\right)} G_{\varepsilon}(x, y) \mu(x) \mu(y) \mathrm{d} x \mathrm{~d} y \leq\left[u_{\varepsilon}^{2}\left(r_{\varepsilon}\right)|\ln \varepsilon|+C \ln |\ln \varepsilon|\right] n(\varepsilon) .
$$

Combining (5.15) and (5.21), and using (5.13), we get the result of Lemma 5.6.

It remains to prove the claim in (5.20).

Proof of (5.20). By Corollary 5.3, we write,

$$
\frac{4}{\varepsilon^{4}} \int_{B\left(a_{i}, \varepsilon\right) \times B\left(a_{i}, \varepsilon\right)}\left|v_{\varepsilon}(x, y)\right| \mathrm{d} x \mathrm{~d} y \leq 4 \pi\left|v_{\varepsilon}\left(a_{i}, a_{i}\right)\right|+\frac{C \varepsilon^{\alpha}}{\eta^{2}},
$$

where $\alpha \in] 0,1\left[\right.$ and $\eta=r_{\varepsilon}-R$. Using our particular choice of $\eta=\mathcal{O}\left(|\ln \varepsilon|^{-1 / 2}\right)$, we deduce that

$$
\frac{1}{n(\varepsilon)} \sum_{i=1}^{n(\varepsilon)} \frac{4}{\varepsilon^{4}} \int_{B\left(a_{i}, \varepsilon\right) \times B\left(a_{i}, \varepsilon\right)}\left|v_{\varepsilon}(x, y)\right| \mathrm{d} x \mathrm{~d} y \leq \frac{4 \pi}{n(\varepsilon)}\left(\sum_{i=1}^{n(\varepsilon)}\left|v_{\varepsilon}\left(a_{i}, a_{i}\right)\right|+o(1)\right) .
$$

Recalling the choice of the points $\left(a_{i}\right)$ in Lemma 5.4 , we see that the right hand side above is uniformly bounded by a constant times $\ln |\ln \varepsilon|$, yielding the result in (5.20).

In the next lemma, we state a decomposition of the energy due to [7].

Lemma 5.7. Consider $(u, A) \in H^{1}(\Omega ; \mathbb{C}) \times H^{1}\left(\Omega ; \mathbb{R}^{2}\right)$ and define

$$
A^{\prime}=A-\frac{H}{u_{\varepsilon}^{2}} \nabla^{\perp} h_{\varepsilon}
$$


where $u_{\varepsilon}$ and $h_{\varepsilon}$ are introduced in Theorem 2.1 and (3.4) respectively. Then we have the decomposition of the energy,

$$
\begin{aligned}
\mathcal{F}_{\varepsilon, H}(u, A)= & H^{2} J_{0}(\varepsilon)+\int_{\Omega}\left(u_{\varepsilon}^{2}\left|\left(\nabla-i A^{\prime}\right) u\right|^{2}+\left|\operatorname{curl} A^{\prime}\right|^{2}+\frac{1}{\varepsilon^{2}} u_{\varepsilon}^{4}\left(1-|u|^{2}\right)^{2}\right) \mathrm{d} x \\
& +2 H \int_{\Omega}\left(h_{\varepsilon}-1\right)\left[\operatorname{curl}\left(A^{\prime}+\left(i u, \nabla_{A^{\prime}} u\right)\right)\right] \mathrm{d} x \\
& +H^{2} \int_{\Omega} \frac{1}{u_{\varepsilon}^{2}}\left(|u|^{2}-1\right)\left|\nabla h_{\varepsilon}\right|^{2} \mathrm{~d} x
\end{aligned}
$$

Here, the functional $\mathcal{F}_{\varepsilon, H}$ and the energy $J_{0}(\varepsilon)$ are introduced in $(2.22)$ and $(3.2)$ respectively.

Proof of Proposition 5.1. Let $(\psi, A)$ be the test configuration constructed in (5.8)-(5.11), and put $\varphi=\frac{\psi}{u_{\varepsilon}}$. By Lemma 2.7 , it is sufficient to establish the upper bound

$$
\mathcal{F}_{\varepsilon, H}(\varphi, A) \leq H^{2} J_{0}(\varepsilon)+\left(C_{*}-\lambda\right)(\ln |\ln \varepsilon|)^{2} .
$$

By the construction of $\varphi$ and Theorem 2.1, we get,

$$
\frac{1}{\varepsilon^{2}} \int_{\Omega} u_{\varepsilon}^{2}(1-|\varphi|)^{2} \mathrm{~d} x=\mathcal{O}(1)
$$

By Lemma 3.3, we have

$$
\int_{\Omega} \frac{1}{u_{\varepsilon}^{2}}\left(|\varphi|^{2}-1\right)\left|\nabla h_{\varepsilon}\right|^{2} \mathrm{~d} x \leq C \varepsilon^{2}
$$

Let $\mu\left(\varphi, A^{\prime}\right)=\operatorname{curl}\left(A^{\prime}+\left(i \varphi, \nabla_{A^{\prime}} \varphi\right)\right)$. Notice that

$$
\mu\left(\varphi, A^{\prime}\right)=\left\{\begin{array}{l}
0 \quad \text { in } \Omega \backslash \bigcup_{i} B\left(a_{i}, 2 \varepsilon\right) \\
\mu+\mu_{r}\left(\varphi, A^{\prime}\right) \quad \text { in } \bigcup_{i} B\left(a_{i}, 2 \varepsilon\right)
\end{array}\right.
$$

where $\mu$ is the measure defined in (5.7) and

$$
\mu_{r}\left(\varphi, A^{\prime}\right)=-\left(|\varphi|^{2}-1\right) \operatorname{div}\left(\frac{1}{u_{\varepsilon}^{2}} \nabla h^{\prime}\right)+\left(\nabla^{\perp}|\varphi|^{2}\right) \cdot(\nabla \phi-A)
$$

Using the definition of $\mu$ and Lemma 3.3, we write,

$$
\begin{aligned}
2 H \int_{\Omega}\left(h_{\varepsilon}-1\right)\left[\operatorname{curl}\left(A^{\prime}+\left(i \varphi, \nabla_{A^{\prime}} \varphi\right)\right)\right] \mathrm{d} x= & 2 H\left(h_{\varepsilon}\left(r_{\varepsilon}\right)-1\right) \int_{\Omega} \mu\left(\varphi, A^{\prime}\right) \mathrm{d} x \\
& +2 H \int_{\Omega}\left[h_{\varepsilon}(x)-h_{\varepsilon}\left(r_{\varepsilon}\right)\right] \mu\left(\varphi, A^{\prime}\right) \mathrm{d} x \\
\leq & 4 \pi n(\varepsilon)\left(h_{\varepsilon}\left(r_{\varepsilon}\right)-1\right) H \\
& +2 H\left(h_{\varepsilon}\left(r_{\varepsilon}\right)-1\right) \int_{\Omega} \mu_{r}\left(\varphi, A^{\prime}\right) \mathrm{d} x+C \varepsilon \int_{\Omega}\left|\mu\left(\varphi, A^{\prime}\right)\right| \mathrm{d} x
\end{aligned}
$$


Since $|\varphi|=1$ on $\partial B\left(a_{i}, 2 \varepsilon\right)$, an integration by parts yields

$$
\begin{aligned}
\int_{B\left(a_{i}, 2 \varepsilon\right)} \mu_{r}\left(\varphi, A^{\prime}\right) \mathrm{d} x & =\int_{B\left(a_{i}, 2 \varepsilon\right)}\left[-\left(|\varphi|^{2}-1\right) \operatorname{div}\left(\frac{1}{u_{\varepsilon}^{2}} \nabla h^{\prime}\right)+\left(\nabla^{\perp}\left(|\varphi|^{2}-1\right)\right) \cdot \frac{\nabla^{\perp} h^{\prime}}{u_{\varepsilon}^{2}} h^{\prime}\right] \mathrm{d} x \\
& =\int_{B\left(a_{i}, 2 \varepsilon\right)}\left[-\left(|\varphi|^{2}-1\right) \operatorname{div}\left(\frac{1}{u_{\varepsilon}^{2}} \nabla h^{\prime}\right)+\left(|\varphi|^{2}-1\right) \operatorname{div}\left(\frac{1}{u_{\varepsilon}^{2}} \nabla h^{\prime}\right) h^{\prime}\right] \mathrm{d} x \\
& =0
\end{aligned}
$$

On the other hand, using the definitions of $\mu$ and $\varphi$, and Cauchy-Schwarz inequality, we write,

$$
\begin{aligned}
\int_{B\left(a_{i}, 2 \varepsilon\right)}\left|\mu\left(\varphi, A^{\prime}\right)\right| \mathrm{d} x & \leq \int_{B\left(a_{i}, 2 \varepsilon\right)}\left(\mu+2\left|h^{\prime}\right|+\frac{C}{\varepsilon}\left|\nabla h^{\prime}\right|\right) \mathrm{d} x \\
& \leq 2 \pi+C\left(\int_{B\left(a_{i}, 2 \varepsilon\right)}\left(\left|\nabla h^{\prime}\right|^{2}+\left|h^{\prime}\right|^{2}\right) \mathrm{d} x\right)^{1 / 2} .
\end{aligned}
$$

Therefore, (5.22) becomes, for a new constant $C>0$,

$$
\begin{aligned}
2 H \int_{\Omega}\left(h_{\varepsilon}-1\right)\left[\operatorname{curl}\left(A^{\prime}+\left(i \varphi, \nabla_{A^{\prime}} \varphi\right)\right)\right] \mathrm{d} x \leq & 4 \pi n(\varepsilon)\left(h_{\varepsilon}\left(r_{\varepsilon}\right)-1\right) H \\
& +C \varepsilon\left[\left(\int_{\Omega}\left(\left|\nabla h^{\prime}\right|^{2}+\left|h^{\prime}\right|^{2}\right) \mathrm{d} x\right)^{1 / 2}+n(\varepsilon) H\right] .
\end{aligned}
$$

Thanks to Lemma 5.7, we get

$$
\mathcal{F}_{\varepsilon, H}(\varphi, A) \leq H^{2} J_{0}(\varepsilon)+(1+C \varepsilon) \int_{\Omega}\left(\frac{1}{u_{\varepsilon}^{2}}\left|\nabla h^{\prime}\right|^{2}+\left|h^{\prime}-1\right|^{2}\right) \mathrm{d} x+4 \pi n(\varepsilon)\left(h_{\varepsilon}\left(r_{\varepsilon}\right)-1\right) H+\mathcal{O}(\varepsilon n(\varepsilon) H) .
$$

We recall that the magnetic field satisfies $H=k_{\varepsilon}|\ln \varepsilon|+\lambda \ln |\ln \varepsilon|$, and we apply Lemma 5.6 to deduce the upper bound,

$$
\begin{aligned}
\mathcal{F}_{\varepsilon, H}(\varphi, A) \leq & H^{2} J_{0}(\varepsilon)+2 \pi n(\varepsilon)\left(1-h_{\varepsilon}\left(r_{\varepsilon}\right)\right)\left[\left(\frac{u_{\varepsilon}^{2}\left(r_{\varepsilon}\right)}{1-h_{\varepsilon}\left(r_{\varepsilon}\right)}-2 k_{\varepsilon}\right)|\ln \varepsilon|\right. \\
& \left.+C \frac{n(\varepsilon)+\ln |\ln \varepsilon|}{1-h_{\varepsilon}\left(r_{\varepsilon}\right)}-\lambda \ln |\ln \varepsilon|\right]+o\left(n(\varepsilon)^{2}+n(\varepsilon) \sqrt{\varepsilon}\right) .
\end{aligned}
$$

Recall the definition of $r_{\varepsilon}=R+\frac{\ln |\ln \varepsilon|}{|\ln \varepsilon|}$. Thanks to Lemma 3.3 and Theorem 3.4, we get:

$$
\left|\frac{u_{\varepsilon}^{2}\left(r_{\varepsilon}\right)}{1-h_{\varepsilon}\left(r_{\varepsilon}\right)}-2 k_{\varepsilon}\right| \leq \widetilde{C} \frac{\ln |\ln \varepsilon|}{|\ln \varepsilon|}
$$

Thus, when choosing $C_{*}>2 C+\widetilde{C}$ and $n(\varepsilon)=[\ln |\ln \varepsilon|]([\cdot]$ denotes the largest integer less than $\cdot)$, the upper bound (5.23) becomes,

$$
\mathcal{F}_{\varepsilon, H}(\varphi, A) \leq H^{2} J_{0}(\varepsilon)+\left(C_{*}-\lambda\right)(\ln |\ln \varepsilon|)^{2},
$$

thus achieving the proof of Proposition 5.1. 


\section{Proof of MAIN TheOREMS}

\subsection{Proof of Theorem $\mathbf{1 . 1}$}

Theorem 4.2 provides us with a family of vortex balls $\left(B\left(a_{i}, r_{i}\right)\right)_{i}$. In particular, when the lower bound of Corollary 4.3 is matched with the upper bound (4.1), permits us to deduce,

$$
0 \geq 2 \pi a \sum_{d_{i}>0}\left(|\ln \varepsilon|-C \ln |\ln \varepsilon|-k_{\varepsilon}^{-1} H\right)\left|d_{i}\right|+2 \pi \sum_{d_{i} \leq 0}(a|\ln \varepsilon|-C \ln |\ln \varepsilon|)\left|d_{i}\right| .
$$

Taking $\lambda_{*}<-C$ and $\lambda \leq \lambda_{*}$, we deduce that $\sum_{i}\left|d_{i}\right|=0$ whenever the magnetic field satisfies $H<k_{\varepsilon}|\ln \varepsilon|+$ $\lambda \ln |\ln \varepsilon|$. The energy decomposition of Lemma 5.7, together with point (4) of Proposition 4.1, yield now the estimate

$$
\frac{1}{\varepsilon^{2}} \int_{\Omega}\left(1-|\varphi|^{2}\right) \mathrm{d} x \ll 1
$$

which when combined with Lemma 2.9 gives the desired result, $|\varphi| \geq \frac{1}{2}$ in $\bar{\Omega}$.

Now, when $\lambda \geq \lambda_{*}$, the properties (a)-(c) of Theorem 1.1 are consequences of Theorem 4.2 and Lemma 4.4, which give in particular the upper bound on the total degree $\sum_{i}\left|d_{i}\right| \leq C \ln |\ln \varepsilon|$.

Assume now that $H=k_{\varepsilon}|\ln \varepsilon|+\lambda \ln |\ln \varepsilon|$, with $\lambda>0$. When the lower bound of Corollary 4.3

$$
\mathcal{F}_{\varepsilon, H}(\varphi, A) \geq H^{2} J_{0}(\varepsilon)+2 \pi a \sum_{d_{i}>0}\left(|\ln \varepsilon|-C \ln |\ln \varepsilon|-k_{\varepsilon}^{-1} H\right) d_{i}+2 \pi \sum_{d_{i} \leq 0}(a|\ln \varepsilon|-C \ln |\ln \varepsilon|)\left|d_{i}\right|
$$

is matched with the upper bound of Proposition 5.1, we deduce that

$$
2 \pi a \sum_{d_{i}>0}(C-\lambda) \ln |\ln \varepsilon| d_{i} \leq\left(C_{*}-\lambda\right)(\ln |\ln \varepsilon|)^{2} .
$$

Taking $\lambda>\max \left(C_{*}, C\right)$, we deduce the desired lower bound on the total degree

$$
\sum_{i}\left|d_{i}\right| \geq \sum_{d_{i}>0} d_{i} \geq c \ln |\ln \varepsilon|
$$

This achieves the proof of Theorem 1.1.

\subsection{Proof of Theorem $\mathbf{1 . 2}$}

Let $(\psi, A)$ be a minimizer of (1.1) such that $|\psi|>0$ in $\bar{\Omega}$. Then all the degrees $\left(d_{i}\right)$ in Theorem 4.2 are null:

$$
d_{i}=0 \quad \forall i .
$$

It results now from the upper bound $\mathcal{F}_{\varepsilon, H}(\varphi, A) \leq H^{2} J_{0}(\varepsilon)$, the lower bound of Theorem 4.2 and the energy decomposition of Lemma 5.7:

$$
\int_{\Omega}\left(\left|\left(\nabla-i A^{\prime}\right) \varphi\right|^{2}+\frac{1}{2 \varepsilon^{2}}\left(1-|\varphi|^{2}\right)^{2}+\left|\operatorname{curl} A-H h_{\varepsilon}\right|^{2}\right) \mathrm{d} x \ll 1 \quad(\varepsilon \rightarrow 0),
$$

where $A^{\prime}=A-\frac{H}{u_{\varepsilon}^{2}} \nabla^{\perp} h_{\varepsilon}$.

From this estimate and the G-L equation satisfied by $\varphi$, we are able to prove ( $c f$. [22], Lem. 6.4) the following estimate

$$
\varepsilon\left\|\left(\nabla-i A^{\prime}\right) \varphi\right\|_{H^{1}\left(S_{1}\right)} \ll 1 \quad(\varepsilon \rightarrow 0) .
$$


Consequently, the trace theorem yields

$$
\varepsilon\left\|n(x) \cdot\left(\nabla-i A^{\prime}\right) \varphi\right\|_{L^{2}\left(\partial S_{1}\right)} \ll 1 \quad(\varepsilon \rightarrow 0) .
$$

Since the functions $h_{\varepsilon}$ and $u_{\varepsilon}$ are radial, we have

$$
n(x) \cdot\left(\nabla-i A^{\prime}\right) \varphi=n(x) \cdot(\nabla-i A) \varphi .
$$

Let us also notice that

$$
\left|n(x) \cdot\left[\frac{(\nabla-i A) \psi}{\psi}-\frac{\nabla u_{\varepsilon}}{u_{\varepsilon}}\right]\right|=\frac{1}{|\varphi|}\left|n(x) \cdot\left(\nabla-i A^{\prime}\right) \varphi\right| .
$$

On the other hand, since $\frac{1}{\varepsilon^{2}} \int_{\Omega}\left(1-|\varphi|^{2}\right)^{2} \mathrm{~d} x \ll 1$, Lemma 2.9 yields that $|\varphi| \geq \frac{1}{2}$ in $\bar{\Omega}$. Therefore, we deduce that

$$
\varepsilon\left\|n(x) \cdot\left[\frac{(\nabla-i A) \psi}{\psi}-\frac{\nabla u_{\varepsilon}}{u_{\varepsilon}}\right]\right\|_{L^{2}\left(\partial S_{1}\right)} \leq 2 \varepsilon\left\|n(x) \cdot\left(\nabla-i A^{\prime}\right) \varphi\right\|_{L^{2}\left(\partial S_{1}\right)} \ll 1 .
$$

Now, invoking Theorem 2.4, we conclude the result of Theorem 1.2, with $\gamma(a)$ given in (2.8).

\subsection{The regime $a>1$}

Let us sum up what we know in this case. Let us introduce the following Ginzburg-Landau functional analyzed in [8]

$$
H^{1}(\Omega ; \mathbb{C}) \ni u \mapsto F_{\varepsilon}(u)=\int_{\Omega}\left(|\nabla u|^{2}+\frac{1}{2 \varepsilon^{2}}\left(1-|u|^{2}\right)^{2}\right) \mathrm{d} x
$$

Let us also recall the definition of the function $\xi_{\varepsilon}: \Omega \rightarrow \mathbb{R}_{-}$introduced in (3.16). We recall also the set $\Lambda_{\varepsilon}=\left\{x \in \bar{\Omega}:\left|\xi_{\varepsilon}(x)\right|=\max _{\bar{\Omega}}\left|\xi_{\varepsilon}\right|\right\}$ which governs the location of the vortices of a minimizer of (1.1).

Now, the result of Lemma 4.4 permits to prove the existence of a constant $\mathcal{M}>0$ such that (see [28], Sect. 3)

$$
F_{\varepsilon}(\varphi)<\mathcal{M}|\ln \varepsilon|
$$

where $\varphi=\frac{\psi}{u_{\varepsilon}}$ and $(\psi, A)$ always denote a minimizer of (1.1).

On the other hand, the result of Theorem 3.4 states that

$$
\Lambda_{\varepsilon}=\{0\}, \quad \xi_{\varepsilon}^{\prime \prime}(0)>0 .
$$

The estimate (6.2) is the basis on which the analysis in [30] is build-up. It permits to prove an expression of the first critical field:

$$
H_{C_{1}}=k_{\varepsilon}|\ln \varepsilon|+k_{1, \varepsilon}
$$

where $k_{\varepsilon}$ is given by (4.6) and $k_{1, \varepsilon}=\mathcal{O}(1)$. If $H_{C_{1}}+k<H<H_{C_{1}}+\mathcal{O}(1), k>0$, then a minimizer $(\psi, A)$ of (1.1) has a finite number of vortices, each of degree 1 , and localized near the center of the disc $\Omega=D(0,1)$. Furthermore, it is proved that if more than one vortex exists, distinct vortices will tend, after normalization, to distinct points in $\mathbb{R}^{2}$.

The results in (6.3) are the basis to build-up the analysis of [31], which permits to obtain a sequence of critical fields. We point out that in order to adapt the analysis of [31], we need to remember that in every compact subset $K$ of $D(0, R)$, the function $u_{\varepsilon}$ converges to 1 exponentially fast in $C^{2}(K)$.

We define the normalized energy:

$$
w_{\varepsilon, n}: \mathbb{R}^{n} \ni\left(x_{1}, x_{2}, \ldots, x_{n}\right) \mapsto-2 \pi \sum_{i \neq j} \ln \left|x_{i}-x_{j}\right|+2 \pi \xi_{\varepsilon}^{\prime \prime}(0) \sum_{i=1}^{n}\left|x_{i}\right|^{2} .
$$


The analysis of [31] yields that, if the magnetic field satisfies

$$
H=k_{\varepsilon}(|\ln \varepsilon|+\delta \ln |\ln \varepsilon|), \quad n-1<\delta<n, \quad n \in \mathbb{N},
$$

then a minimizer $(\psi, A)$ of $(1.1)$ has $n$-vortices $\left(x_{i}(\varepsilon)\right)_{i=1}^{n}$, each of degree 1 , and such that, when putting $\widetilde{x}_{i}(\varepsilon)=x_{i}(\varepsilon) \sqrt{H}$, then the configuration $\left(\widetilde{x}_{i}(\varepsilon)\right)_{i=1}^{n}$ is localized near a minimizer of the renormalized energy $w_{\varepsilon, n}$. Furthermore, the following expansion of the energy holds as $\varepsilon \rightarrow 0$ :

$$
\begin{aligned}
\mathcal{F}_{\varepsilon, H}(\varphi, A)= & H^{2} J_{0}(\varepsilon)-2 \pi n\left(|\ln \varepsilon|-\frac{H}{k_{\varepsilon}}\right)+\pi\left(n^{2}-n\right) \ln H \\
& +w_{\varepsilon, n}\left(\widetilde{x}_{1}(\varepsilon), \ldots, \widetilde{x}_{n}(\varepsilon)\right)+Q_{n}+o(1),
\end{aligned}
$$

where $Q_{n}$ is an explicit constant depending only on $n$.

\section{REFERENCES}

[1] A. Aftalion, E. Sandier and S. Serfaty, Pinning phenomena in the Ginzburg-Landau model of superconductivity. J. Math. Pures Appl. 80 (2001) 339-372.

[2] A. Aftalion, S. Alama and L. Bronsard, Giant vortex and the breakdown of strong pinning in a rotating Bose-Einstein condensate. Arch. Rational Mech. Anal. 178 (2005) 247-286.

[3] S. Alama and L. Bronsard, Pinning effects and their breakdown for a Ginzburg-Landau model with normal inclusions. J. Math. Phys. 46 (2005) 095102.

[4] S. Alama and L. Bronsard, Vortices and pinning effects for the Ginzburg-Landau model in multiply connected domains. Comm. Pure Appl. Math. LIX (2006) 0036-0070.

[5] N. André, P. Baumann and D. Phillips, Vortex pinning with bounded fields for the Ginzburg-Landau equation. Ann. Inst. H. Poincaré Anal. Non Linéaire 20 (2003) 705-729.

[6] H. Aydi and A. Kachmar, Magnetic vortices for a Ginzburg-Landau type energy with discontinuous constraint. II. Comm. Pure Appl. Anal. 8 (2009) 977-998.

[7] F. Béthuel and T. Rivière, Vortices for a variational problem related to superconductivity. Ann. Inst. H. Poincaré Anal. Non Linéaire 12 (1995) 243-303.

[8] F. Béthuel, H. Brezis and F. Hélein, Ginzburg-Landau vortices. Birkhäuser, Boston-Basel-Berlin (1994).

[9] S.J. Chapman and G. Richardson, Vortex pinning by inhomogenities in type II superconductors. Phys. D 108 (1997) $397-407$.

[10] S.J. Chapman, Q. Du and M.D. Gunzburger, A Ginzburg Landau type model of superconducting/normal junctions including Josephson junctions. European J. Appl. Math. 6 (1996) 97-114.

[11] P.G. de Gennes, Superconductivity of metals and alloys. Benjamin (1966).

[12] Q. Du, M. Gunzburger and J. Peterson, Analysis and approximation of the Ginzburg-Landau model of superconductivity. SIAM Reviews 34 (1992) 529-560.

[13] H.J. Fink and W.C.H. Joiner, Surface nucleation and boundary conditions in superconductors. Phys. Rev. Lett. 23 (1969) 120.

[14] T. Giorgi, Superconductors surrounded by normal materials. Proc. Roy. Soc. Edinburgh Sec. A 135 (2005) 331-356.

[15] T. Giorgi and D. Phillips, The breakdown of superconductivity due to strong fields for the Ginzburg-Landau model. SIAM J. Math. Anal. 30 (1999) 341-359.

[16] J.O. Indekeu, F. Clarysse and E. Montevecchi, Wetting phase transition and superconductivity: The role of suface enhancement of the order parameter in the GL theory. Procceding of the NATO ASI, Albena, Bulgaria (1998).

[17] A. Kachmar, On the ground state energy for a magnetic Schrödinger operator and the effect of the de Gennes boundary condition. J. Math. Phys. 47 (2006) 072106.

[18] A. Kachmar, On the perfect superconducting state for a generalized Ginzburg-Landau equation. Asymptot. Anal. 54 (2007) $125-164$.

[19] A. Kachmar, On the stability of normal states for a generalized Ginzburg-Landau model. Asymptot. Anal. 55 (2007) $145-201$.

[20] A. Kachmar, Weyl asymptotics for magnetic Schrödinger opertors and de Gennes' boundary condition. Rev. Math. Phys. 20 (2008) 901-932.

[21] A. Kachmar, Magnetic Ginzburg-Landau functional with discontinuous constraint. C. R. Math. Acad. Sci. Paris 346 (2008) $297-300$.

[22] A. Kachmar, Limiting jump conditions for Josephson junctions in Ginzburg-Landau theory. Differential Integral Equations 21 (2008) 95-130.

[23] L. Lassoued and P. Mironescu, Ginzburg-Landau type energy with discontinuous constraint. J. Anal. Math. 77 (1999) 1-26.

[24] K. Lu and X.-B. Pan, Ginzburg-Landau equation with de Gennes boundary condition. J. Diff. Equ. 129 (1996) 136-165. 
[25] N.G. Meyers, An $L^{p}$ estimate for the gradient of solutions of second order elliptic equations. Ann. Sc. Norm. Sup. Pisa 17 (1963) 189-206.

[26] E. Montevecchi and J.O. Indekeu, Effects of confinement and surface enhancement on superconductivity. Phys. Rev. B 62 (2000) 661-666.

[27] J. Rubinstein, Six lectures in superconductivity, in Boundaries, Interfaces and Transitions (Banff, AB, 1995), CRM Proc., Lecture Notes 13, Amer. Math. Soc., Providence, RI (1998) 163-184.

[28] E. Sandier and S. Serfaty, Ginzburg-Landau minimizers near the first critical field have bounded vorticity. Calc. Var. Partial Differ. Equ. 17 (2003) 17-28.

[29] E. Sandier and S. Serfaty, Vortices for the magnetic Ginzburg-Landau model, Progress in Nonlinear Differential Equations and their Applications 70. Birkhäuser Boston (2007).

[30] S. Serfaty, Local minimizers for the Ginzburg-Landau energy near critical magnetic field. I. Commun. Contemp. Math. 1 (1999) 213-254.

[31] S. Serfaty, Local minimizers for the Ginzburg-Landau energy near critical magnetic field. II. Commun. Contemp. Math. 1 (1999) 295-333.

[32] I.M. Sigal and F. Ting, Pinning of magnetic vortices by an external potential. St. Petresburg Math. J. 16 (2005) $211-236$.

[33] G. Stampacchia, Équations elliptiques du second ordre à coefficients discontinus. Séminaire de Mathématiques Supérieures No. 16 (Été, 1965), Les Presses de l’Université de Montréal, Montréal, Québec (1966) 326 p. 